\title{
Dentoalveolar Injuries and Wiring Techniques
}

\author{
Omkar Anand Shetye
}

\subsection{Definition}

Dentoalveolar fracture is defined as a fracture in the bone surrounding the teeth without any extension to the basal bones of the maxilla or mandible $[1,2]$.

\subsection{Incidence}

Dentoalveolar injuries commonly occur in the age group of 8-12 years. They generally involve teeth, soft tissues, and/or associated bone. Traumatic dental injuries account for a total of $92 \%$ of maxillofacial injuries of which $92 \%$ involves only the soft tissues and $8 \%$ involve the maxillofacial bones [2].

\subsection{Introduction}

Trauma is the foremost etiological factor that leads to a loss of significant number of teeth annually. The recent advancements in the field of preventive dentistry as well as the development of myriad evidence-based techniques to restore the lost form and function of teeth have made it possible for the dental surgeon to successfully facilitate the improvement in the quality of life of trauma-afflicted patients. Time elapsed post-trauma plays a major factor in determining the outcome of the intervention. Apart from reestablishing the premorbid position of the traumatized tooth in its socket, the goal of the treatment is directed toward achieving the pre-traumatic occlusion and intra-arch contour [3].

Electronic Supplementary Material The online version of this chapter (https://doi.org/10.1007/978-981-15-1346-6_50) contains supplementary material, which is available to authorized users.

\section{O. A. Shetye $(\square)$}

Department of Oral and Maxillofacial Surgery, Goa Dental College and Hospital, Bambolim, Goa, India

\subsection{Diagnosis and Treatment Planning}

The foundation of diagnosis and management of traumatic dental injuries solely lies on the pillars of a detailed patient history and a clinical examination. When, Where, and How forms the "Trauma Triad" of history taking in traumatic dental injuries [4].

When-Time interval between injury and treatment may significantly affect the prognosis for cases of pulp exposure, displacement, and avulsion type of injuries.

Where-The place of injury. The chances of the injury getting infected on a playground compared to an injury that has occurred at home in relatively cleaner surroundings. The place of injury has medico-legal repercussions if the assault or accident has occurred at the place of work. Depending on the cleanliness of the wound, the clinician can also consider protection against tetanus.

How-This may give an indication of concomitant injuries like soft tissue injuries secondary to the trauma [5].

History of immediate local measures employed to reduce the severity of injury helps in eliciting information regarding the original condition of the injured area [6].

History of any systemic disorders that may interfere with treatment or post-operative healing including a history of tetanus vaccination has to be obtained before starting the treatment [7].

"Outside-in" examination (examining the facial structure in three concentric rings paying more attention to the innermost ring) helps in achieving the desired management protocol. Level of consciousness should be assessed prior to the clinical examination [6].

The potential for aspiration, airway compromise, and neurosensory deficit warrants the need for a thorough evaluation of all maxillofacial injuries before managing dental injuries [3]. 
In a patient with missing tooth fragment or prosthesis, a chest radiograph and abdominal radiograph may be helpful in locating the missing structures and their management. Owing to its anatomic position, the right bronchus is often the site of foreign body dislodgement $[3,6]$.

\subsection{Clinical Examination}

Clinical examination of the patient has to be divided into the following subsets [8]:

- Extra-oral soft tissue

- Intra-oral soft tissue

- Jaws and alveolar bone

- Teeth (displacement and mobility)

- Percussion and pulp testing [3]

\subsubsection{Extra-oral}

Asymmetry of the face following trauma could be due to injury to the facial skeleton or swelling. Areas of ecchymosis and hematoma may indicate fracture of the underlying osseous structure. Laceration, contusion, and abrasion of the overlying skin are common with dentoalveolar injuries (Figs. 50.1, 50.2, and 50.3). Mild antiseptic soap should be used to clean the extra-oral abrasions or a saline wash can be used while being careful to not inoculate injury site further with debris or foreign body $[4,8]$.

\subsubsection{Intra-oral}

Buccal mucosa lacerations should raise suspicion for a Stensen's duct injury. The lips, floor of the mouth, and tongue are areas which are at a risk of being penetrated or sites for secondary injuries [3].

\subsubsection{Jaws and Alveolar Bone}

Bimanual palpation of the maxillary and mandibular dentition and evaluation of occlusion should reveal areas of discrepancy or mobility. The direction of dislocation of the apex of primary tooth should be diagnosed since these teeth are in close approximation to the succedaneous teeth. Palpating from the vestibular approach makes this diagnosis easier. Sublingual ecchymosis/hematoma (Coleman's sign) at the floor of the

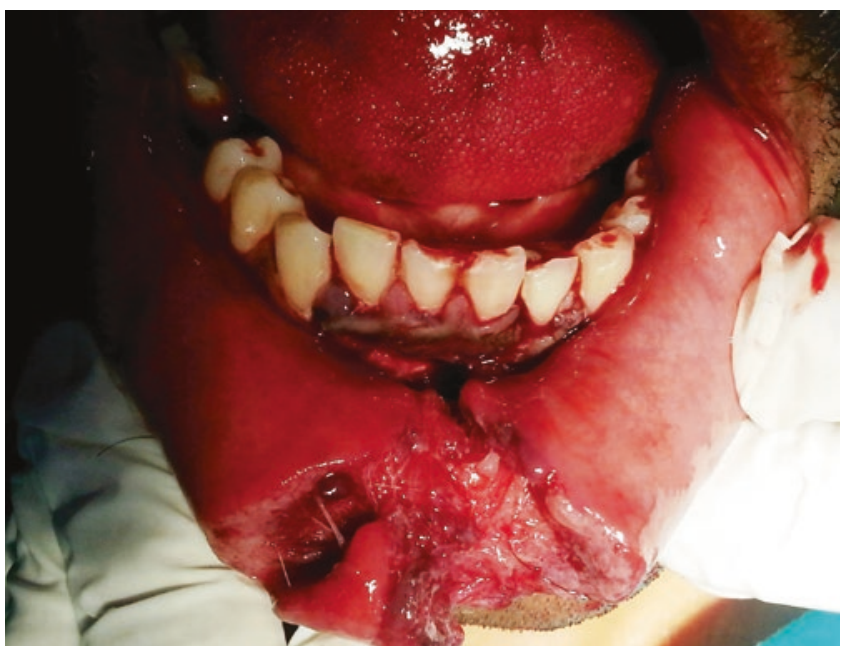

CAssociation of Oral and Maxillofacial Surgeons of India

Fig. 50.1 Mandibular anterior lingually displaced dentoalveolar fracture with degloving vestibular wound and ragged lip lacerations

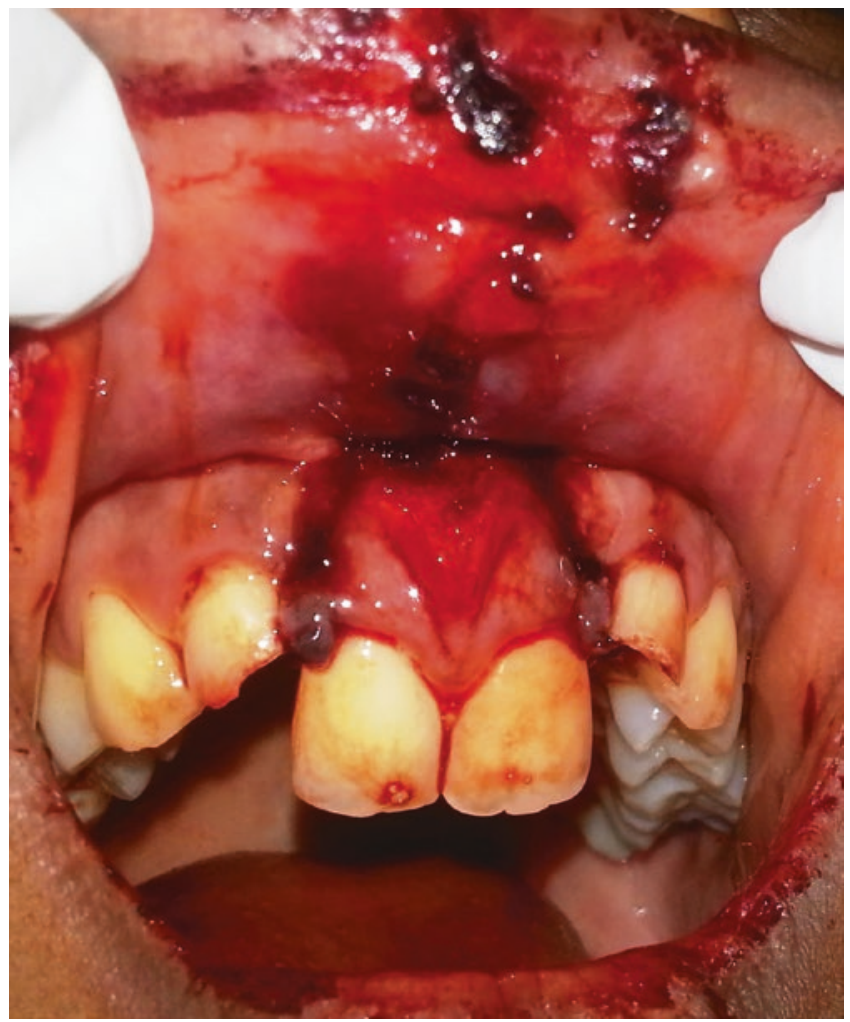

CAssociation of Oral and Maxillofacial Surgeons of India

Fig. 50.2 Maxillary anterior palatally displaced and extruded dentoalveolar fracture with associated upper lip injury. Note incisal fractures of the upper lateral incisors (see also Fig. 50.8)

mouth is suggestive of underlying mandibular fractures. Step deformity, crepitation, malocclusion, and gingival lacerations raise suspicion for possible bone defects [7, 9]. 


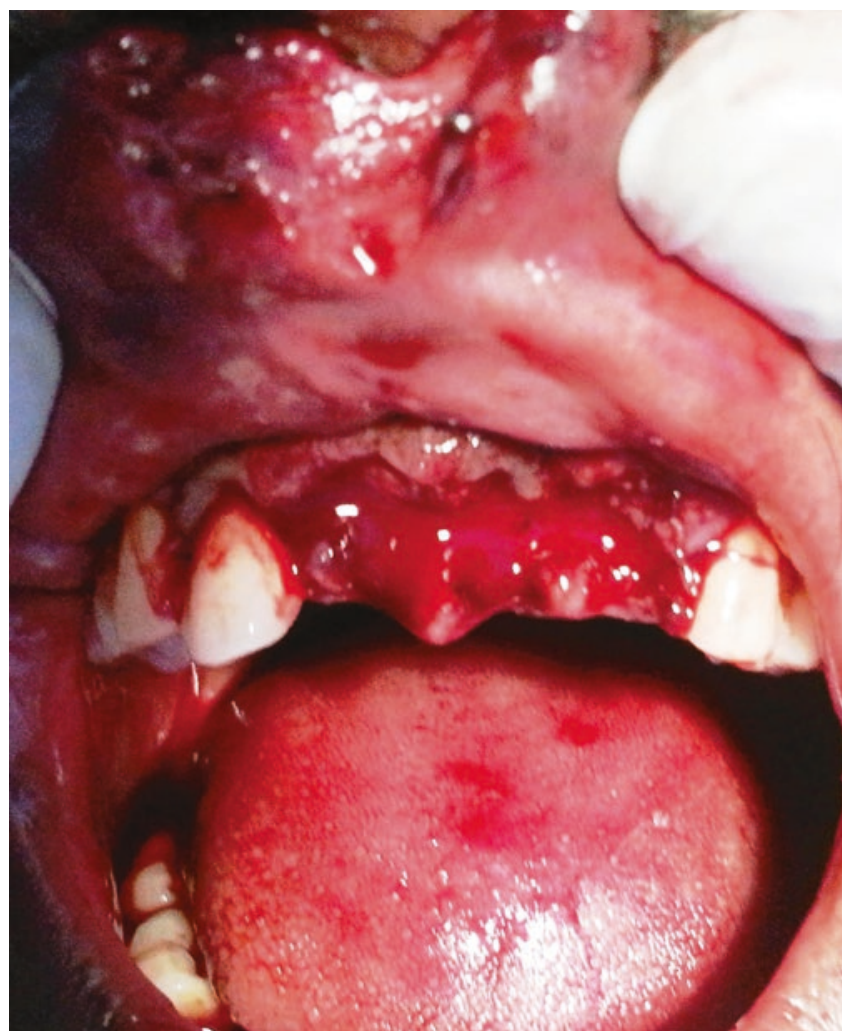

CAssociation of Oral and Maxillofacial Surgeons of India

Fig. 50.3 Maxillary anterior teeth complete avulsion with associated upper lip injury

\subsubsection{Percussion and Pulp Testing}

Percussion test is performed with the end of the handle of an examination mirror. Pain to percussion of a tooth is indicative of damage to the periodontal ligament. Dullness to percussion in one or several adjacent teeth can be indicative of partially luxated teeth or en bloc fracture of tooth or alveolar bone. The percussion test should be started on a non-injured tooth to ensure a reliable response through visual analogue scale $[6,8]$.

To evaluate the tooth injury thoroughly, pulp vitality is a must. A positive response indicates that the pulp is alive, whereas a negative response indicates that the pulp is dead. Following injury, the tooth may be in a state of shock and may give a false response. Hence pulp testing should also be performed during the subsequent visits to acknowledge the change in response [5].

Electric vitality testing depends on a number of factors including the stage of eruption of tooth, the presence of a restoration or decay, and the ability to isolate the tooth and keep the area clear of blood and saliva [8].

Laser Doppler flowmetry (LDF) is a relatively new pulp testing apparatus. It employs a laser beam directed at the

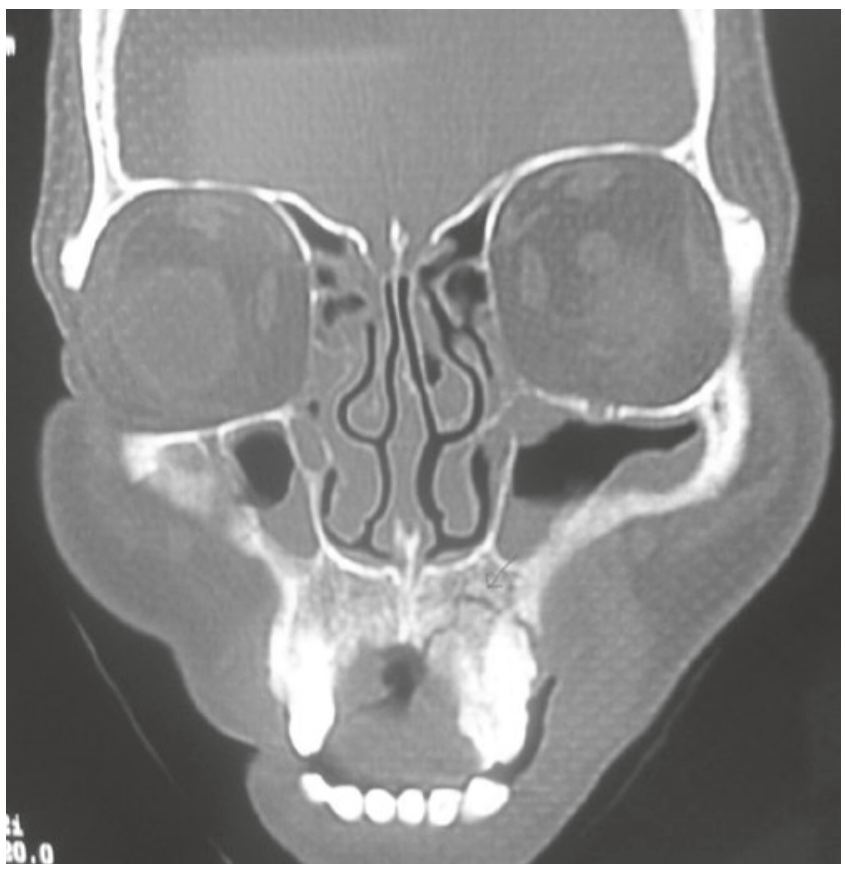

CAssociation of Oral and Maxillofacial Surgeons of India

Fig. 50.4 Coronal CT image showing left maxillary high dentoalveolar fracture

coronal-labial aspect of the pulp which is scattered by pulp blood cells that in turn produce a Doppler frequency shift. The fraction of light scattered back is detected and processed to elicit a signal. The basic theory is that the pulp revascularization process can be monitored. Studies have shown that in cases in which electrometric tests were negative and LDF displayed vascular perfusion, the LDF accuracy of pulp vitality reached $100 \%$ [3].

All the teeth should be tested for abnormal mobility both horizontally and vertically. It should be remembered that primary teeth undergoing physiologic root resorption and therefore always exhibit a certain degree of mobility. The typical sign of an alveolar fracture is the movement of adjacent teeth when the mobility of a single tooth is tested [6].

\subsection{Radiographic Examination}

Radiographic techniques are available to evaluate dentoalveolar trauma.

Radiographic examination is essential to determine damage to underlying structures and should include intraoral periapical (IOPA), occlusal, panoramic radiographs (OPG), and cone-beam computed tomography (CBCT) imaging [3, 4].

If conventional CT imaging is done as part of the trauma series, fine axial and coronal cuts will also show the dentoalveolar injuries (Fig. 50.4) 
IOPA radiograph provides vital information about root fracture and dislocation of teeth. At times it is difficult to adequately evaluate a fracture on an IOPA radiograph, due to variations in tube-tooth-film geometry. Post-treatment radiographs can confirm the proper repositioning of avulsed or luxated tooth into alveolar bone.

Occlusal radiographs provide a larger field of view, and a steep occlusal exposure is of special value in the diagnosis of root fracture and lateral luxations with displacement of crown [8].

OPG is a useful screening tool and can demonstrate fractures of the mandible and maxilla as well as fractures of alveolar ridges and teeth.

Radiographic evaluation of foreign bodies within soft tissues of the lips and cheeks is done by taking radiographs with the film placed labial to the alveolus. A reduced radiographic exposure time (approximately $1 / 3 \mathrm{rd}$ of the normal) is used.

CBCT scanning is extremely useful in diagnosis of maxillofacial, alveolar bone, and teeth fractures with the advantage of high-resolution three-dimensional images with low radiation.

The etiology of pediatric and adult dentoalveolar injuries were shown in Box 50.1 and Box 50.2 respectively. Box 50.3 and Box 50.4 represents the classification of alveolar bone fracture and classification of alveolar bone fracture based on location and displacement respectively.

Box 50.1: Etiology of Pediatric Dentoalveolar Injuries

Pediatric patients [9]

- Bicycle injuries

- Falls

- Sports injuries

- Car crashes

- Child abuse

- Iatrogenic injuries(dental extractions, endoscopy, oral intubation)

- Industrial accidents
Box 50.2: Etiology of Adult Dentoalveolar Injuries

Adult patients

- Motor vehicular accidents

- Interpersonal violence

- Sports injuries(contact injuries)

- Falls

- Work-related trauma

Box 50.3: Classification of Alveolar Bone Fracture:

Traumatic alveolar bone [9]

Type I: Areas surrounding a single tooth

Type II: Entire dentoalveolar segment dislocation

Box 50.4 Alveolar fracture can be classified by their specific location and movement of displacement [10]

Class I: Edentulous segment fracture

Class II: Little or any displacement of fractured dentulous segment

Class III: Moderate to severe displacement of fractured dentulous segment

Class IV: Multiple fracture lines with combination of fracture of the dentulous segment 


\section{Box 50.5: Classification of Dental Injuries and Surrounding Structure}

\section{Classification based on level of involvement [11]}

1. Fracture involving enamel

2. Fracture involving enamel and dentin

3. Fracture involving enamel, dentin and pulp

4. Fracture involving enamel, dentin, cementum

5. Crown/ root fracture

6. Luxation of tooth

7. Intrusion and extrusion (Fig. 50.5a,b) (Box 50.6)

8. Avulsion (Fig. 50.5c) (Boxes 50.7 and 50.8)
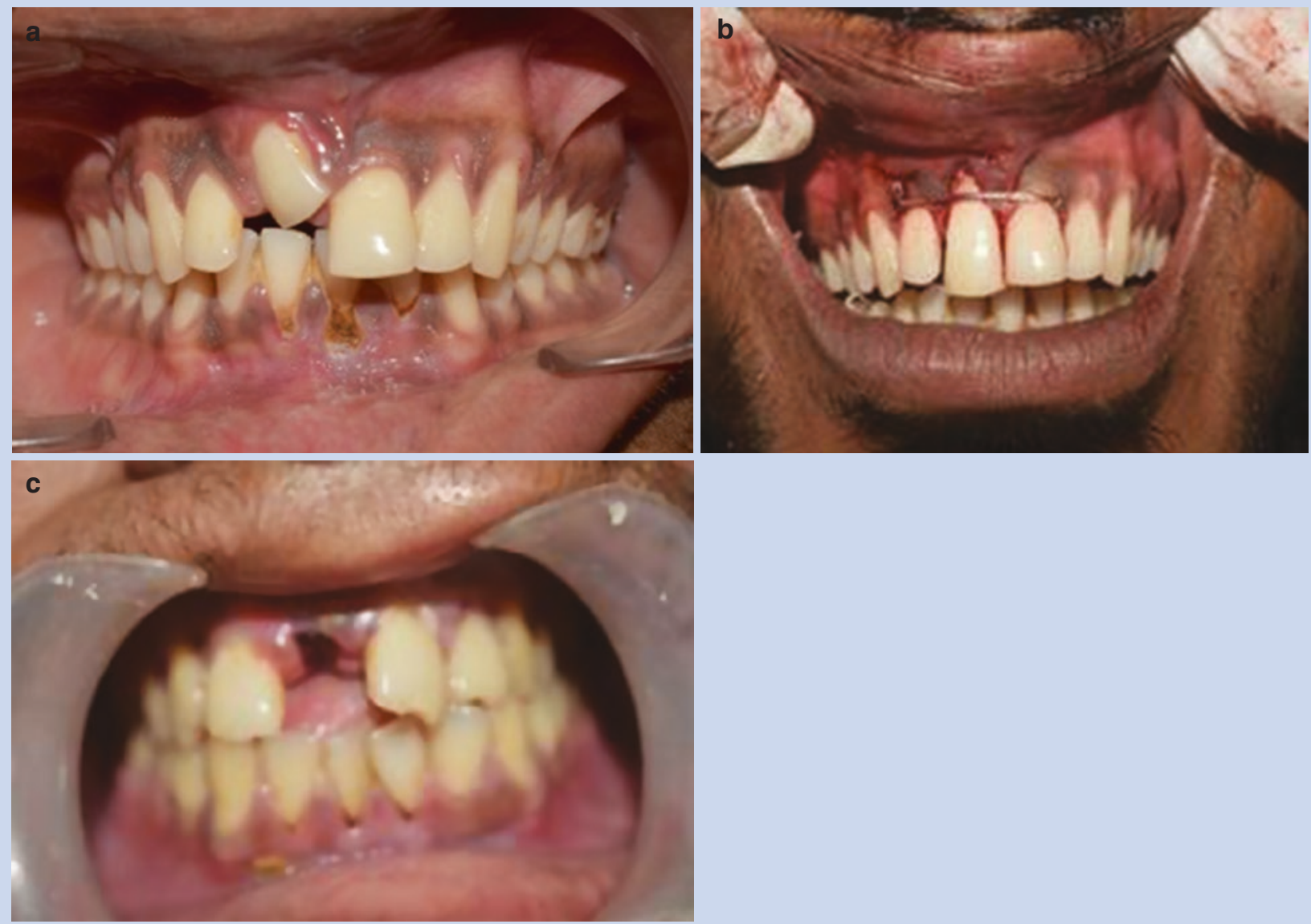

CAssociation of Oral and Maxillofacial Surgeons of India

Fig. 50.5 (a-c) Clinical images showing tooth (a) intrusion, (b) extrusion, and (c) avulsion 


\section{Box 50.6: Management of Intruded Tooth (Fig. 50.6)}

Diangelis et al. [12, 13]

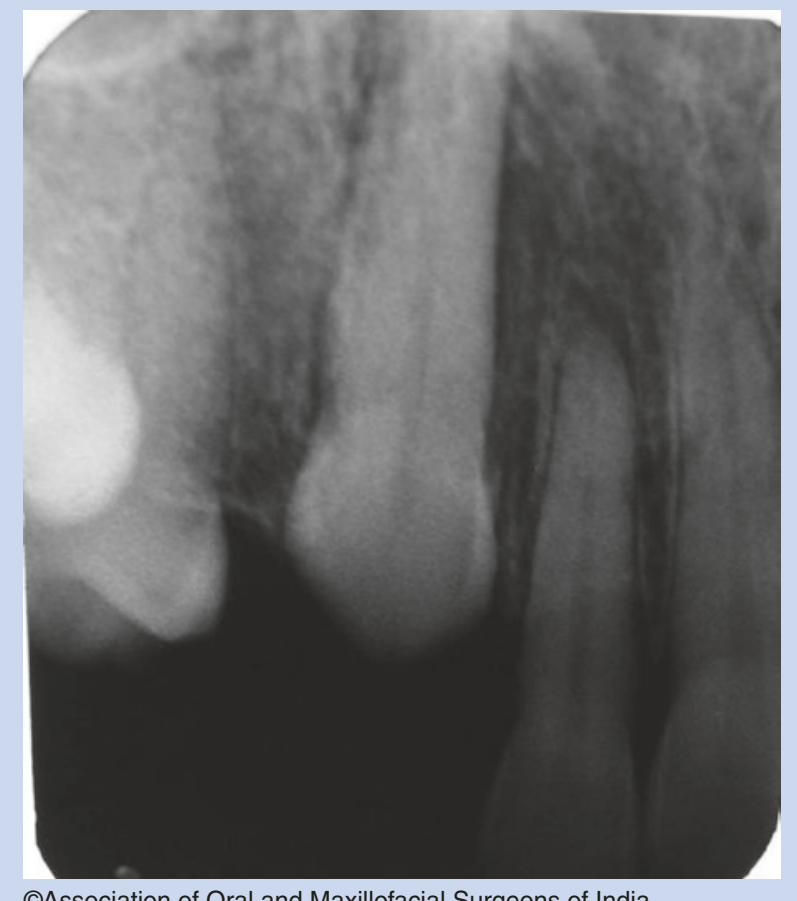

(C)Association of Oral and Maxillofacial Surgeons of India

Fig. 50.6 IOPA showing an intruded upper right canine

\section{Permanent Teeth}

1. In closed apex

- Intrusion $<3 \mathrm{~mm}$-allow eruption

- Intrusion between 3 and $7 \mathrm{~mm}$-surgical or orthodontic repositioning

- Intrusion $>7 \mathrm{~m} \mathrm{~m}$

(a) Surgical extrusion with flexible splint for 4-8 weeks

(b) $\mathrm{RCT}$ using $\mathrm{Ca}(\mathrm{OH})_{2}$ medicament 2-3 weeks after surgical extrusion

2. In open apex

(a) Wait till the tooth erupts for 3 months

(b) Place an orthodontic extruding appliance

\section{Primary Teeth}

Treatment is debatable

Immediate extraction of the involved tooth

or

More conservative approach

\section{Box 50.7: Principle of Avulsion}

- Primary should not be replanted

- Permanent teeth should be replanted as soon as possible and stabilized for 7-10 days up to 2 weeks $[14,15]$.

\section{Box 50.8: Primary Management of Avulsion by the \\ Patient}

What the patient can do:

- To hold the tooth by its crown and not to touch the root surface.

- To rinse the tooth immediately with saliva, tap water or saline solution and reimplant the tooth.

- If the patient cannot replace the tooth, it should be held in the buccal vestibule (only if patient is fully conscious).

- Gentle irrigation with saline or gauze soaked with saline may be employed to remove blood clots or foreign objects from the surface of the root.

What the patient should not do:

- No effort should be made to mechanically cleanse the root of the tooth because this would damage the remaining periodontal ligament.

Transportation media for avulsed teeth include

1. Viaspan: a specialized tissue storage media used when transporting liver for transplantation.

2. Hanks balanced salt solution: both hanks solution and viaspan are physiologic with compatible $\mathrm{pH}$ and osmolality. The relative availability and cost-effective hanks solution makes it medium of choice in storage of avulsed teeth.

Commercial products are designed especially for storing avulsed teeth. It has shelf life of 2 years without refrigeration (Box 50.9).

3. Milk: is a readily available medium. It is the medium of choice in the absence of hanks balanced salt solution or viaspan. Milk will only prevent further cellular demise and is used specifically when the teeth has been outside the oral cavity for less than $20 \mathrm{~min}$.
If the tooth cannot be reimplanted within 5 min, it should be stored in a medium that will maintain its vitality and periodontal ligament fibers [14-16]. 


\section{Box 50.9: HBSS Contents}

Contents of Hanks balanced salt solution [19]

- Sodium chloride

- Glucose

- Potassium chloride

- Sodium bicarbonate

- Sodium phosphate

- Calcium chloride

- Magnesium chloride

- Magnesium sulfate

4. Saliva: is an excellent transport medium and is as effective as saline. It does not matter if the tooth is stored in another person's saliva.

5. Tap water: has been commonly recommended storage solution but due its hypotonicity, it leads to rapid cell lysis and increased inflammation on replantation.

6. Contact lens solution: a compilation of information from a study Blomlof et al. [17] indicates that Hanks buffered saline, isotonic saline, and pasteurized bovine milk may be the most favorable known storage media. If none of these are available, human saliva or contact lens solution is acceptable short-term substitute storage liquid.

7. Culture mediums like minimum essential eagles medium and Dulbecco's storage media [18] have been used as they contain amino acids, vitamins, and bicarbonates that help to maintain the vitality of cells.

8. Propolis: a beehive product which has an excellent antiinflammatory and anti-bacterial effect has been used as a storage medium.

9. Green Tea: its polyphenol derivative have antioxidative, anti-carcinogenic, anti-mutagenic, anti-inflammatory, antimicrobial, and antiviral effect.

10. Patients own serum have shown to maintain the vitality of periodontal ligament cells up to $1 \mathrm{~h}$.

Morus rubra (red mulberry), egg white, coconut water, rehydrating solution like Gatorade and ricetral, lens solution, probiotic solutions, saliva officinalis, honey milk, and ascorbic acid have been used as a transportation medium for avulsed tooth.

\subsection{Treatment at the Clinic}

Traumatically avulsed teeth when out of the mouth for a short period should be reimplanted as soon as possible.

\section{Box 50.10: Reagent for Root Surface Therapy}

Agents used for root surface treatment to prevent resorption

- Citric acid

- Doxycycline

- Tetracycline

- Fluorides

- Enamel matrix derivative (emdogain)

- Diphosphonates

After normal reattachment, survival is prolonged. Avulsed teeth which remain out of the mouth for more than $2-3 \mathrm{~h}$ generally resorb rapidly and should not be reimplanted indiscriminately.

The outcome of replantation depends on the stage of root development and extra-oral time. If the avulsed tooth has a closed apex and extra-oral time is less than $60 \mathrm{~min}$ (early replantation), the reimplanted tooth will have the best prognosis. The ideal time to begin root canal treatment is within 10-14 days post-replantation and before splint removal.

There is a statistically significant association between extirpation within 14 days and an increased likelihood of successful periodontal healing and prevention of external inflammatory root resorption.

It is generally agreed that $\mathrm{Ca}(\mathrm{OH})_{2}$ has a beneficial effect in the outcome because of its antibacterial properties, ability to dissolve necrotic tissue, and its ability to prevent or control inflammatory resorption. The anti-inflammatory and antibacterial action may decrease root resorption by directly inhibiting resorptive cells (Box 50.10).

\subsubsection{Delayed Replantation (more than $60 \mathrm{~min}$ )}

Delayed replantation of avulsed tooth with closed apex has a poor long-term prognosis. The periodontal ligament gets necrotic and healing is delayed. The goal of delayed replantation is to promote alveolar bone growth so as to encapsulate the reimplanted tooth. The eventual outcome is ankylosis.

In children below age of 15 years, if ankylosis occurs and when the infra position of the tooth crown is more than $1 \mathrm{~mm}$, decoronation is recommended to preserve the contour of the alveolar bone. 
Root canal treatment (RCT) can be carried out on a tooth prior to reimplantation or it can be carried out within 14 days of reimplantation. The tooth is immersed in $2 \%$ sodium fluoride solution for a minimum of $5 \mathrm{~min}$ up to $20 \mathrm{~min}$.

Avulsed teeth having open apices and extra-oral time less than $60 \mathrm{~min}$ are also reimplanted and stabilized for 7-10 days and up to 2 weeks. The goal of reimplanting developing (immature) teeth in children is to allow for possible revascularization of the tooth pulp.

If there is evidence of the pulpal infection or inflammatory response, the pulp should be removed and $\mathrm{Ca}(\mathrm{OH})_{2}$ placed immediately, aiming to stimulate apexification and halt the inflammatory process.

It is believed that delayed replantation of avulsed teeth with open apices have a poor long-term prognosis. The peri-
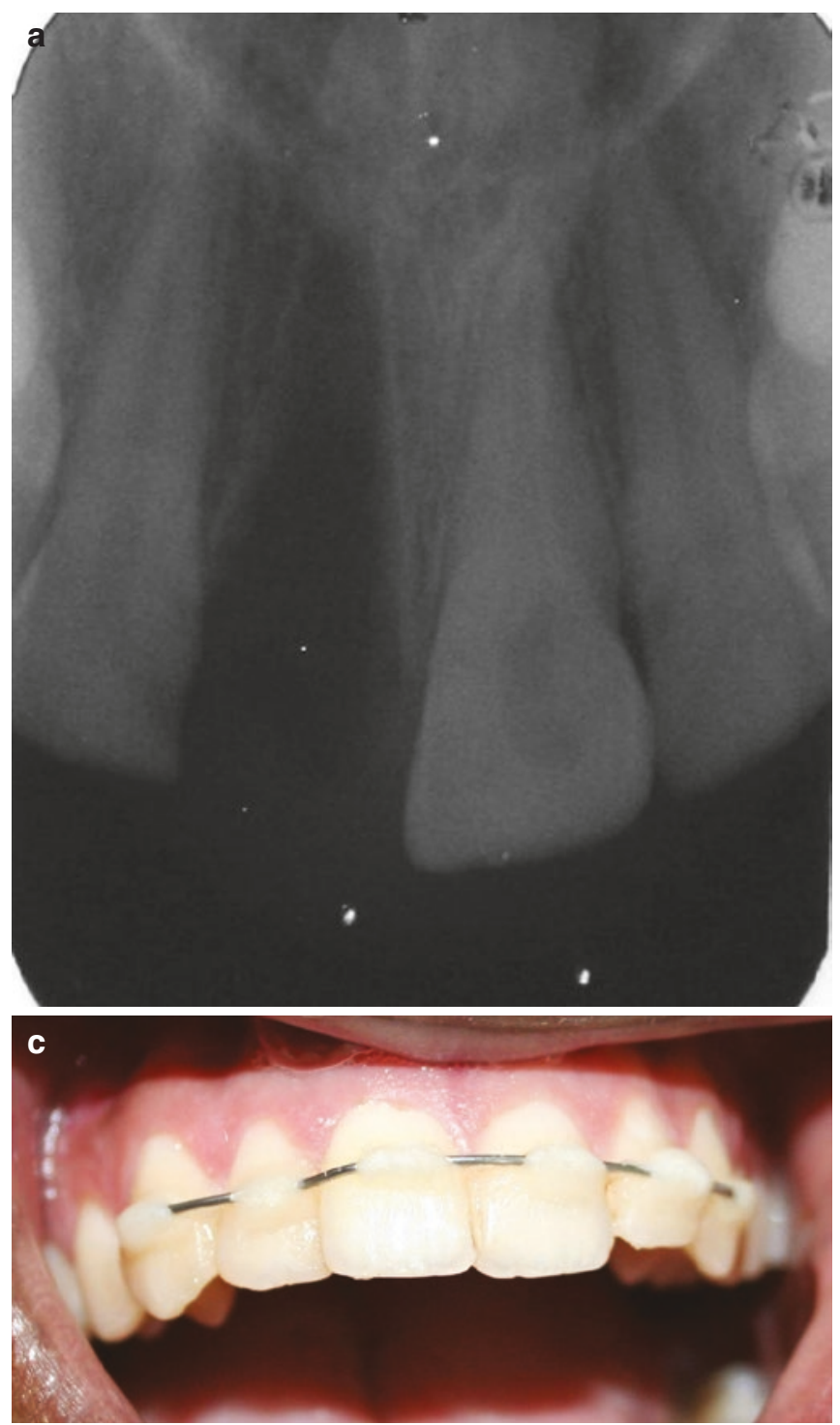

CAssociation of Oral and Maxillofacial Surgeons of India

Fig. 50.7 (a-c) Shows a case of avulsed upper right central incisor in a 24-year-old man, which presented after few hours of avulsion. The tooth was transported in milk by the relatives. A RCT was carried out odontal ligament gets necrotic and is not expected to heal. Osseous replacement, resorption, or ankylosis will occur. Therefore some authors have concluded that such teeth should not be reimplanted.

The recent guidelines recommend replantation to maintain alveolar ridge contour as ankylosed roots get transformed to bone during the remodeling process. RCT can be performed on the tooth prior to replantation through an open apex (Fig. 50.7a-c).

\subsubsection{Stabilization}

Stabilization of an avulsed tooth can be achieved using a variety of materials like wires, arch bar wired to the teeth,

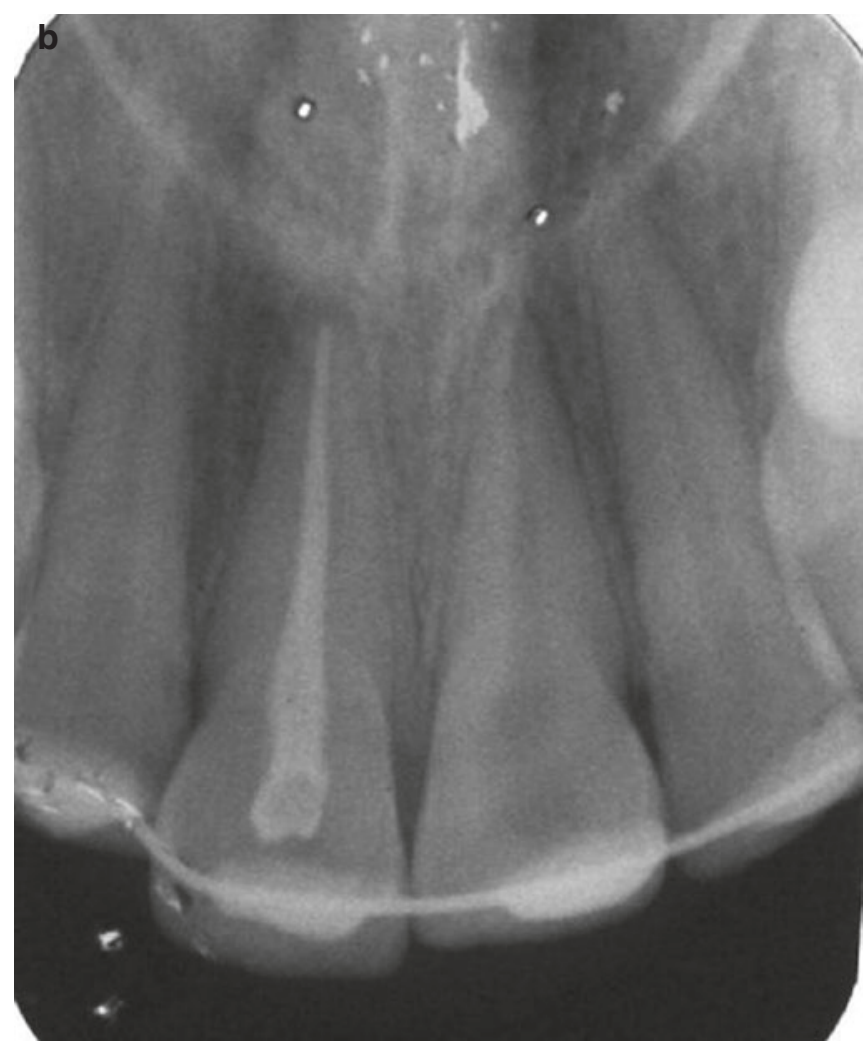

and the tooth reimplanted and stabilized with wire and composite splint (a, IOPA showing avulsed incisor; $\mathbf{b}$, IOPA with tooth reimplanted; $\mathbf{c}$, shows the clinical image after splinting) 
orthodontic band and brackets attached by acid etch technique, combined with orthodontic band and acrylic appliance, or periodontal pack.

They are generally maintained for 7-10 days after replantation to allow gingival reattachment.

Studies by Andreason [20] (1985) have shown that prolonged splinting may be inadvisable because it enhances ankylosis.

The socket should be left undisturbed before replantation. If the alveolar bone has collapsed, a blunt instrument should be inserted carefully into the socket in an attempt to reposition the wall. It should be lightly aspirated if a blood clot is present.

In general, stabilization for reimplanted teeth is required for 7-14 days. The periodontal ligament fibers should have healed sufficiently after the first week to remove the splint. However, the patient should be advised not to bite directly on the reimplanted tooth for 3-4 weeks after injury.

\subsubsection{Technique}

\subsubsection{Wire Acid Etch Composite Splint}

A wire of moderate stiffness (round 0.030 in. stainless steel) is adapted to the facial surface at least one or more stable teeth on either side of the reimplanted teeth.

The physiologic movement imparted to the reimplanted tooth during function is increased when fewer teeth are included in stabilization of the avulsed tooth.

The facial surface of the avulsed and adjacent teeth is acid etched, and wire is cemented to them with composite.

The wire is generally curved along the middle third of the labial surface of the teeth. $90^{\circ}$ terminal bends toward the gingiva are made at the labiodistal angles of the most posterior teeth to be splinted in order to avoid sharp edges and stability. The terminal ends should be about $\mathbf{2} \mathbf{~ m m}$ long to reduce the possibility of lateral displacement of the wire [21, 22].

\begin{tabular}{|c|c|}
\hline Traumatic dental injuries & Splinting period \\
\hline Subluxation & 2 weeks \\
\hline Extrusive luxation & 2 weeks \\
\hline Avulsion & 2 weeks \\
\hline Lateral luxation & 4 weeks \\
\hline Intrusion(if surgically repositioned) & 4-8 weeks \\
\hline Root fracture(middle third) & 4 weeks \\
\hline Root fracture(cervical third) & 4 months \\
\hline
\end{tabular}

\subsubsection{Complications}

1. Pulp necrosis

2. Pulp chamber or root canal obliteration

3. Internal resorption

4. External resorption-three types:

(a) Surface replacement

(b) Replacement resorption (ankylosis)

(c) Soft tissue replacement [23]

\subsection{Alveolar Process Fracture}

Fracture of the alveolar process usually involves the teeth.

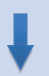

Place the segment into position (closed reduction)

(Fig. 50.8)

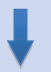

Open reduction/ raise the flap if reduction not possible (Fig. 50.9 a, b)

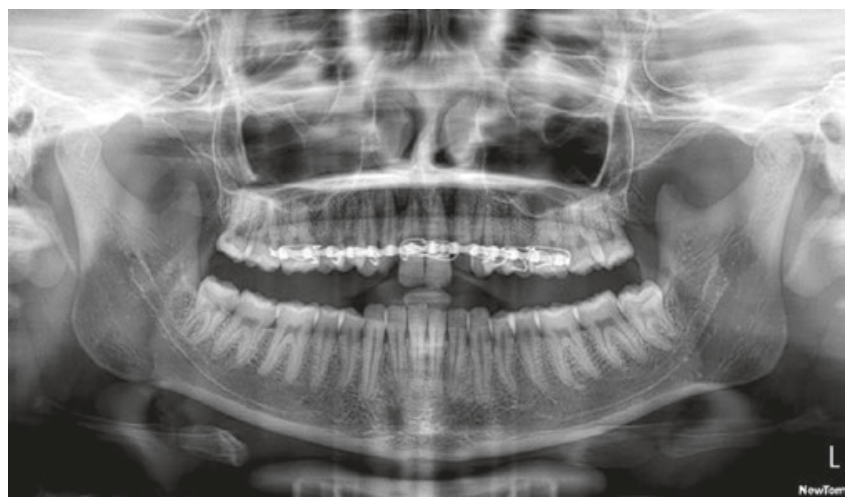

CAssociation of Oral and Maxillofacial Surgeons of India

Fig. 50.8 Shows the case in Fig. 50.2, which has undergone closed reduction and fixation by arch bar. Full intrusion and reduction was not achieved. The occlusal discrepancy was planned to be corrected later by RCT and necessary prosthetic work 

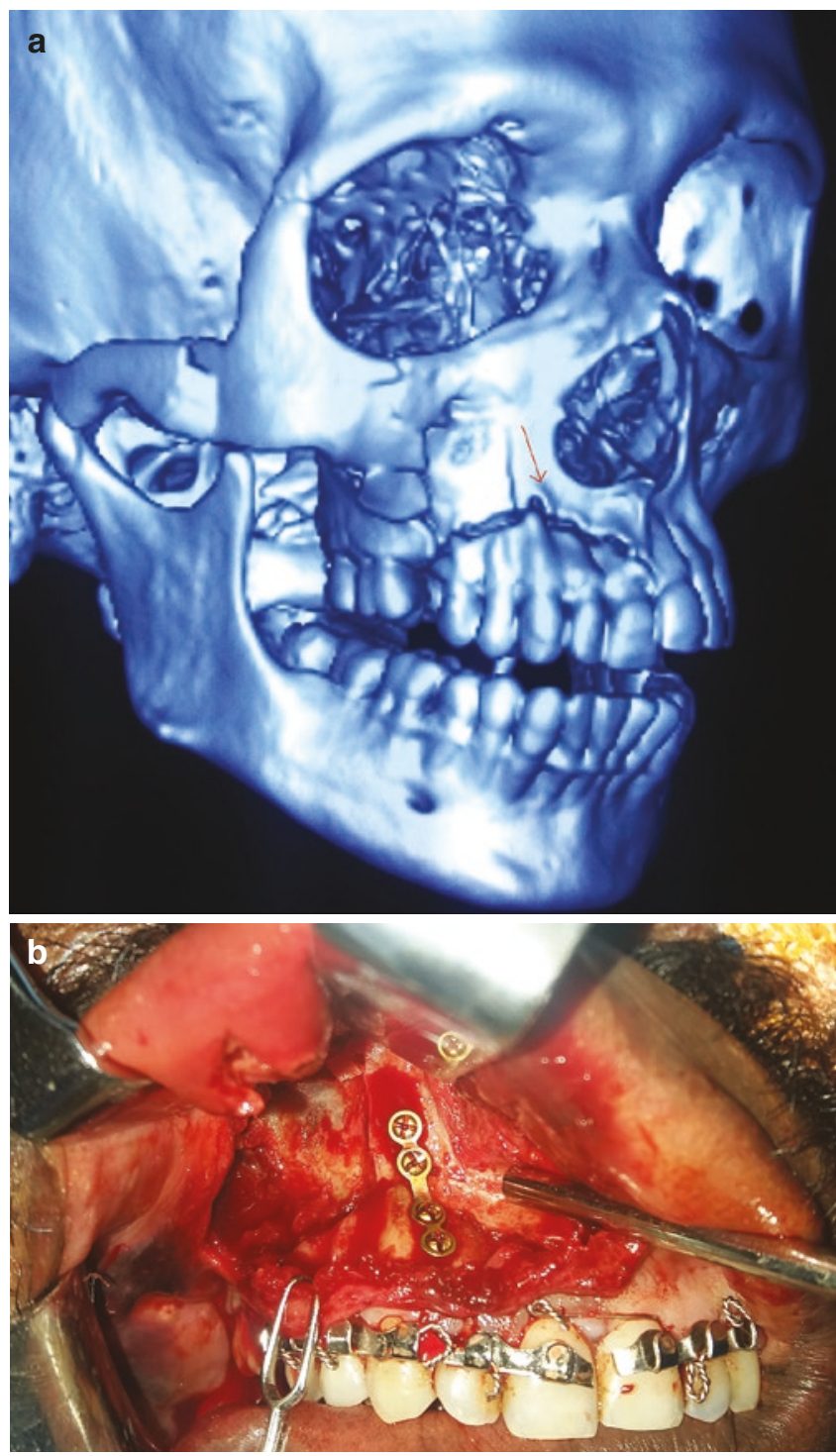

CAssociation of Oral and Maxillofacial Surgeons of India

Fig. 50.9 (a, b) Shows a case of high alveolar process fracture segment in the maxillary right side with associated split between central incisors. Under GA, the extruded displaced alveolar segment was repositioned after placement of split arch bars and bridle wiring between the central incisors. The segment was fixed with mini plate and screws, taking care not to injure the tooth roots. Due to root anatomy, only a single plate was fixed after reduction. Post-operative IMF was given for 2 weeks, for additional stability and to help the healing process

\subsection{Management of Dentoalveolar Fracture}

The goals of treatment should be:
(a) Anatomic reduction
(b) Re establishment of premorbid occlusion
(c) Early return of function
(d) Preservation of premorbid arch form and facial contour [24]

\subsubsection{Treatment Options}

1. Arch bars (Fig. 50.8)

2. Loop wiring

3. Orthodontic bands

4. Acrylic/metallic splints

5. Acid etch composite

Model surgery on the plaster models is performed to recreate the normal anatomic relationship of the fractured segment and allow fabrication of accurate splint [9].

\subsubsection{Specific Treatment Options}

Class I fractures that involve a non-displaced, edentulous alveolar segment often do not require treatment other than a soft diet and observation. If there is concern about the stability of the fracture during the healing process, stabilization may be used.

Class II fractures that involve a displaced dentulous segment and need reduction may require a great deal of force to realign the fractured segment. Posterior fragments are always displaced to the lingual area. Large forceps may be helpful to apply a force suitable to reduce the bony fragment. Reduced fragments are held in place by MMF or splints.

Class III fractures of a moderately or severely displaced dentulous segment may be too difficult to reduce adequately. There may be scant room into which the irregular, displaced segment needs to be replaced. It may be necessary to burr or rasp down the displaced segment or the opening in the remaining bone to successfully reduce the fracture. This is usually accomplished by use of a power drill with a suitable size bur or a fine rhinoplasty rasp. The amount of tissue that is removed should be limited so that the sufficient bone to bone contact remains for bony union as the fracture heals. Maintenance of class III fractures in a reduced fashion may be accomplished with arch bars, MMF, and/or variety of splints.

Class IV fractures that extend into one or more non alveolar fracture lines are usually less challenging than class III fractures because (1) the bone segments are larger, (2) the treatment of the associated fractures gives excellent exposure, and (3) usually no barrier exists to reduction. Plates, screws, arch bars, MMF, and/or variety of splints may be used [7].

\subsubsection{Wiring Techniques}

In MMF, wires are passed interdentally between teeth in both the jaws followed by tightening of these wires together in a cross arch fashion which serves to stabilize the fracture segments. 
Over the period of last century, MMF techniques have evolved dramatically, and more recently, many more easier and effective methods have been introduced like MMF screws, eyelet wiring, and Erich arch bars to name a few. Although it is a quick and efficient way of securing MMF, yet, this technique is criticized for extended periods the patient needs to be closed mouth, difficulty in maintaining oral hygiene, lack or deficient nutritional support via oral route and inability to use this treatment modality in medically compromised patients like epileptics or in immediate post-operative period due to increased risk of aspiration.

\subsubsection{Armamentarium and Principles} (Fig. 50.10)

A soft stainless steel wire of $0.35-0.5 \mathrm{~mm}$ diameter is utilized and pre-stretched to $10 \%$ of its original length to prevent loosening of wire once tightened. Overstretching beyond this point should be avoided as this will make the wire brittle and susceptible to breakage.

\subsubsection{Principles}

Basic principles to be followed are [25]:

1. Continuous tension on the wire while twisting and tightening.

2. Force should be apically directed while tightening.

3. Utilizing a clock wise direction for all tightening/ twisting.

4. Only half a turn to be given at a time at the end.

5. Once the tail is cut, it is turned and placed into the inter-proximal embrasures.

6. Once all the wires are in place, move the finger around the arch bar/wire to check for any sharp edge/margin/ wire ending.

\subsubsection{Technique}

\subsubsection{Bridle Wire $[26,27]$}

First advocated by Hippocrates, bridle wire remains one of the oldest yet a conventional method of treating mandibular fractures. This technique represents a temporary way of stabilizing the fractured segments, preventing them from flaying apart.

Advantages include stabilization of the two fractured segments preventing further damage to the adjacent soft tissues, maintenance of the airway patency (especially advan-

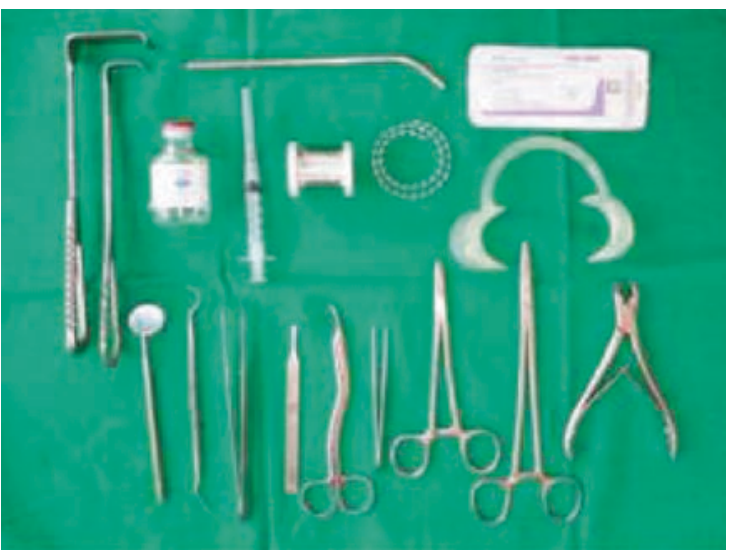

CAssociation of Oral and Maxillofacial Surgeons of India

Fig. 50.10 Armamentarium for wiring

tageous for the fractures of the anterior mandible), decrease pain, and reducing damage to the neurovascular bundle from immobilizing the fractured fragments, thereby preventing gross movements and also decreasing muscle cramping.

\section{Technique}

Following administration of local anesthesia , a 24-26 gauge wire is passed around the neck of the teeth adjacent to the fracture site on either side (Fig. 50.11a). With both ends being secured in the wire holder (Fig. 50.11b), the fractured segments are manually reduced anatomically, and the wire is tightened in a clockwise fashion till further reduction is achieved (Fig. 50.11c), and the fracture is stabilized

\subsubsection{Gilmer's Direct Interdental Wiring} $[26,27]$

This technique represents an easy and fastest method of immobilization.

\section{Technique}

A $15 \mathrm{~cm}$ pre-stretched wire is passed through the interdental embrasure of the tooth on one side from buccal to lingual. This wire will pass around the tooth and emerge out buccally through the interdental embrasure from the other side. Both ends are held together and twisted to achieve a $3 \mathrm{~cm}$ tail (Fig. 50.12a-c). Multiple teeth (at least 5-6) in either jaw are utilized. Following manual fracture reduction and placement of teeth with premorbid occlusion, the tails from opposite arch are twisted together in a cross arch or zigzag fashion to achieve immobilization (Fig. 50.12d). The cut ends of the tail are secured into the interdental spaces. Once all the wires/tails are twisted, complete immobilization is achieved.

The only drawback of this technique is that, in cases of broken /loose wire, all the cross arch wires will have to be removed and redone post placement of twisted wire. 

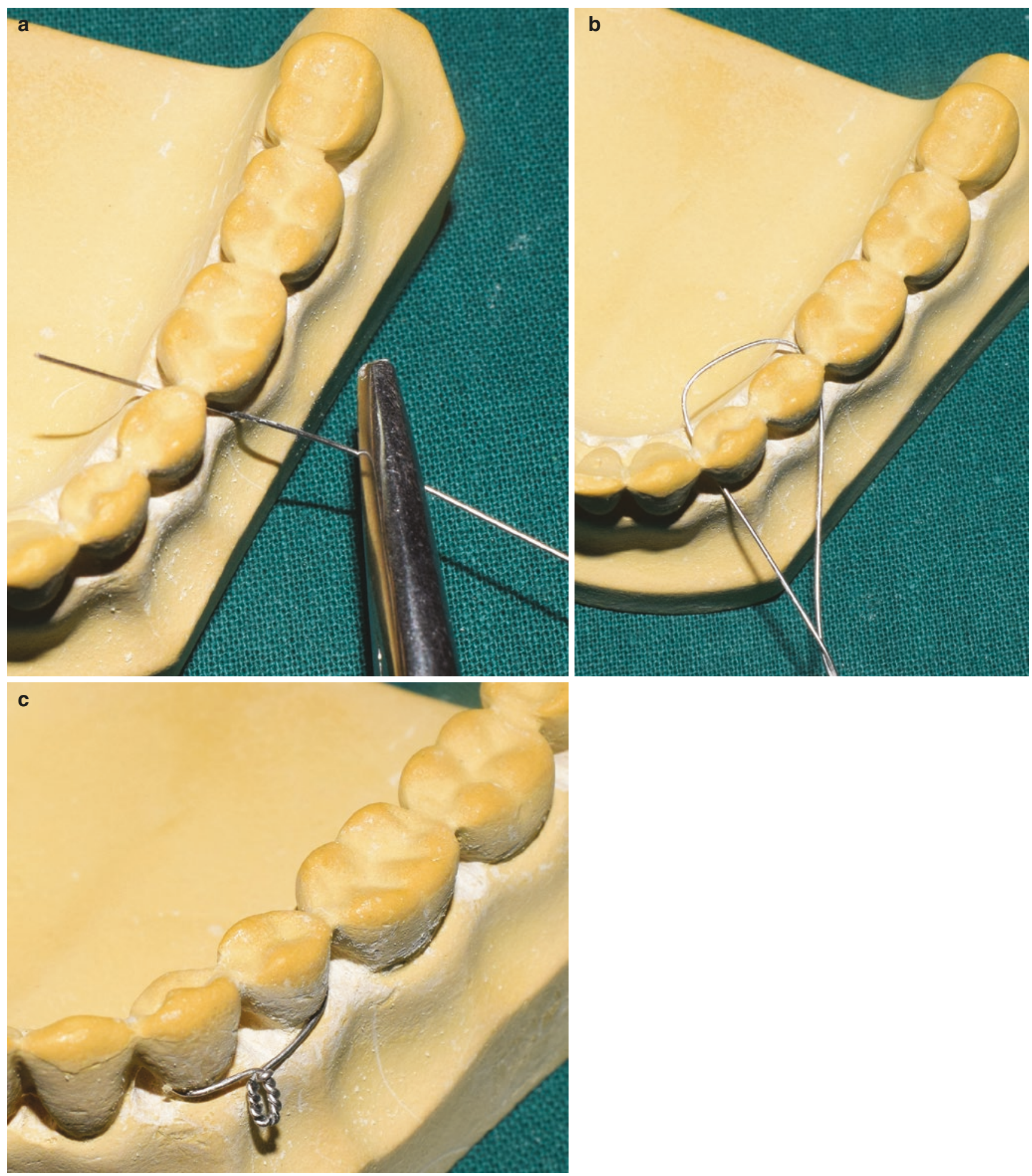

CAssociation of Oral and Maxillofacial Surgeons of India

Fig. 50.11 Shows sequential steps bridel wiring. (a) Passing of the wire through the tooth embrasure. (b) Formation of the wire loop assuming the fracture line is between the two premolars. (c) Twisting of both the ends to stabilize the fracture 

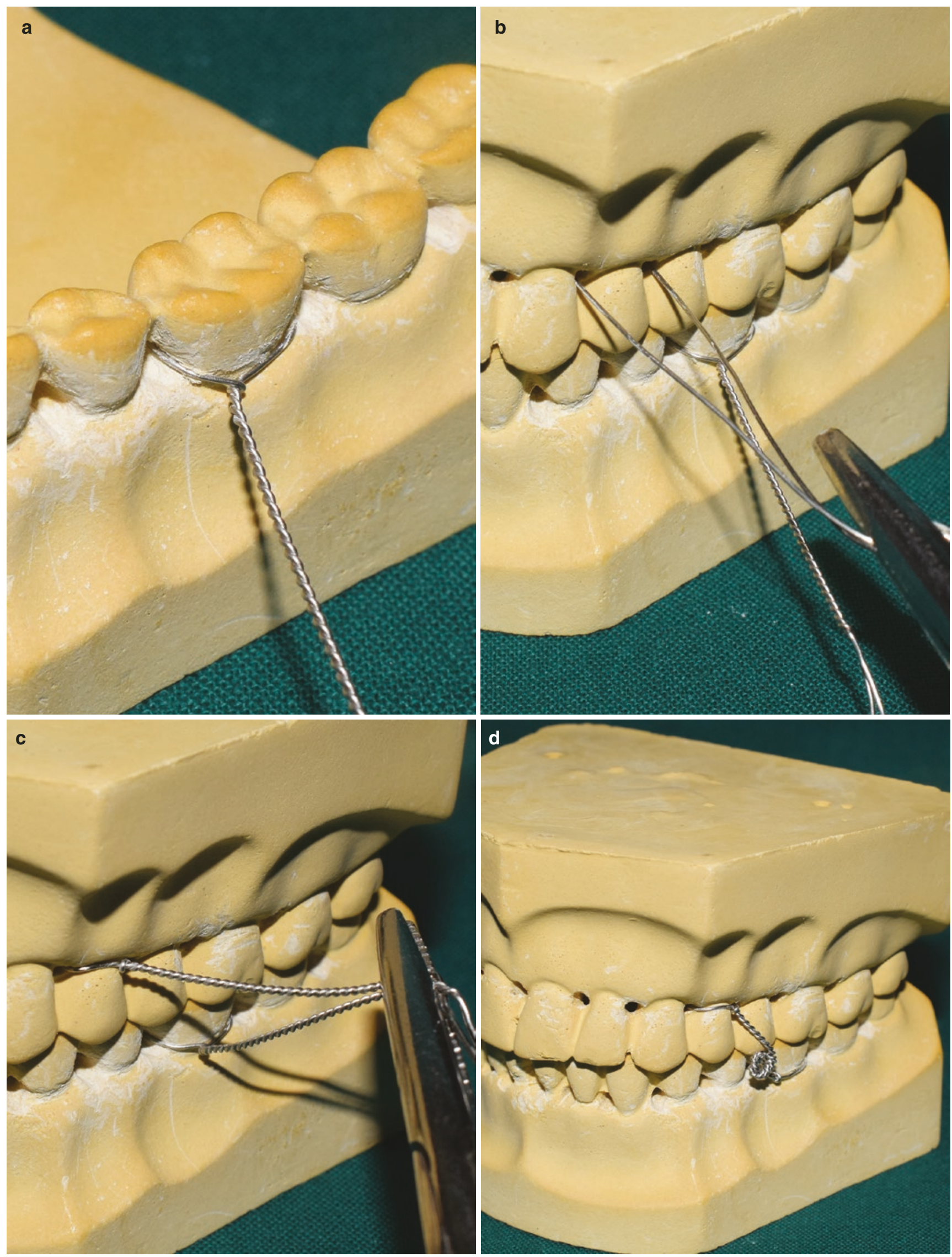

CAssociation of Oral and Maxillofacial Surgeons of India

Fig. 50.12 (a-d) Shows technique of direct Gilmer wiring. Formation of the loop around the tooth in the lower and upper arch followed by twisting both the wires together in order to achieve MMF 


\subsubsection{Interdental Eyelet Wiring (Ivy Loop Method) $[26,27]$ (Video 50.1)}

Yet another easy and quick method of securing MMF was advocated and popularized by Dr. Robert H.Ivy, Philadelphia, USA.

\section{Technique}

A pre-stretched 26 gauge $/ 0.35 \mathrm{~mm}$ diameter wire was made into an Ivy loop utilizing the middle segment of the wire by twisting it around a metallic rod of 3 mm diameter. 3-4 turns are given to achieve adequate stability to the loop. Both the ends of the wire are then cut in an oblique fashion (Fig. 50.13a).

Both the ends are passed through the interdental space to emerge lingually/palatally so that the loop lies buccally (Fig. 50.13b). Following this, one end of the wire is passed from distal interdental space of the same tooth to emerge buccally. This same wire is passed through the loop and secured to the other end of the wire which is passed mesially from the lingual/palatal side to the buccal side of the adjacent tooth side (Fig. 50.13c,d) . Both the ends are tightened (Fig. 50.13e), and the tail end cut short and secured into the dental space mesially to prevent damage to the adjacent soft tissues.

Stability of the MMF is based on the adequate number of Ivy loops placed in both the arches and secured.

In the maxillary arch, loops can be placed between two molars, two premolars, or between lateral incisor and canine or two central incisors. In the mandibular arch, loops are best placed between two molars, two premolars, or between lateral incisor and canine.

\section{Modifications}

1. In 1975, Hallam modified the Ivy loop by addition of another loop proximal to the first for the purpose of ligature/tie wire (Fig. 50.14)

Although an interesting modification yet the disadvantage observed was that due to increase in length and in patients with short vertical dimension, both the proximal loops would meet one another making the placement of MMF extremely difficult.

\section{Clove hitch}

This technique helps in placement of an Ivy loop in cases of missing teeth or a single isolated tooth. It is the easiest way to secure MMF where placement of an arch bar or any other technique of securing MMF is difficult due to lack of supporting teeth.

\section{Technique of Clove Hitch}

The one end of the wire that has an Ivy loop forms a loop around the lone-standing tooth (Fig. 50.15a) . Continue the wire around the tooth forming another loop for the second time (Fig. 50.15b) . The end of the wire is under the first loop
(Fig. 50.15c), and both the ends of the wire are pulled together and tightened (Fig. 50.15d).

\subsubsection{Continuous or Multiple Loop Wiring}

It was first described by Col. Stout in 1943. It represents an easy and simple technique which requires minimal instrumentation. This technique utilizes multiple loops on the buccal surface of the tooth which can be used for both MMF using wire as well as elastic traction for the purpose of fracture reduction. One major advantage of this technique is the use of differential traction force that can be achieved and applied for fracture reduction before securing it with wires for MMF.

\section{Technique}

A $30 \mathrm{~cm}$-long pre-stretched wire is placed on the buccal side of the teeth with the anterior end placed as anteriorly as possible while the posterior end extending up to the last tooth to be used. The wire is passed through the interdental space of the posterior most teeth and emerged through its mesial side. The wire is then passed over the buccal wire and passed back into the same interdental space. A $3 \mathrm{~mm}$ diameter rod can be used parallel to the buccal wire for adequate loop diameter as well as uniformity of the loop (Fig. 50.16a). Once the wire is passed back through the same interdental space, it then emerges out from the mesial interdental space of adjacent tooth (Fig. 50.16b). This continues till the anterior most teeth where both the ends of the wire are twisted together, cut, and placed into the interdental space (Fig. 50.16c,d). Each loop is then twisted, and loops are made of uniform diameter. In cases of securing MMF, the loops are turned toward the occlusal surfaces of the teeth, but in cases of elastic traction, they are turned in the opposite direction toward the gingiva [27].

\section{Modification}

This technique needs modification when some teeth are missing. Herein, once the loop at the mesial end of the tooth adjacent to the missing tooth is formed, both of the free ends of the wires are held together and twisted till the distal end of the next tooth. Once the desired length is achieved, the loop is again formed at the distal end, and the looping continues till the anterior most teeth.

\subsubsection{Risdon's Wiring $[26,27]$}

\section{Indications}

It is an alternative to an arch bar for a fractured mandible that needs fixation.

\section{Technique}

A $25 \mathrm{~cm}$-long wire is passed through the posterior most tooth in the quadrant to emerge bucally, followed by twist- 

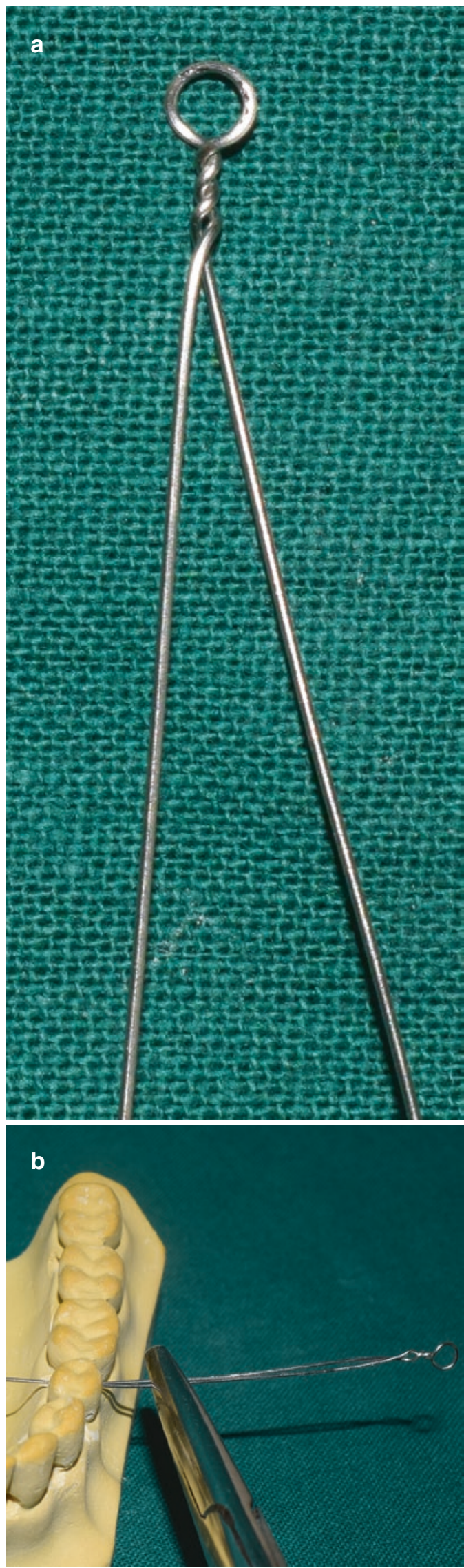

Fig. 50.13 Shows steps in Ivy loop technique. (a) Photograph of an Ivy loop. (b) Both the ends are held together an passed through the tooth embrasure of the premolar and molar. (c) One end passed is passed through the mesial of premolar and other through the distal of

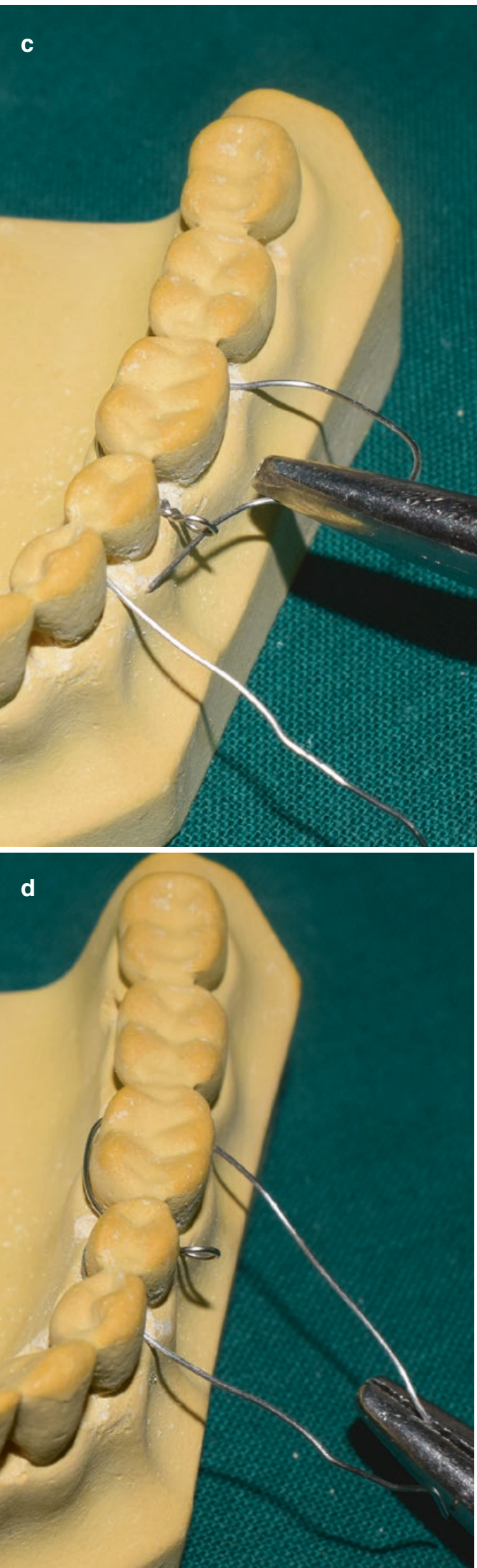

molar to emerge buccally. (d) Distal end is passed through the loop on the buccal side to emerge mesially. (e) Both the ends of the wire are held together and twisted 


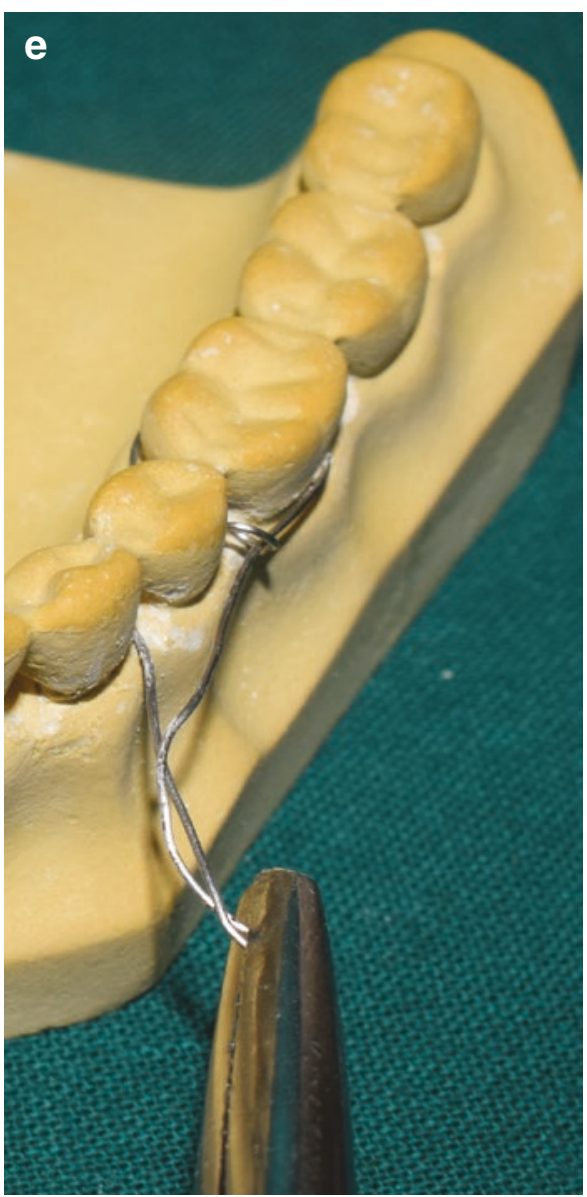

CAssociation of Oral and Maxillofacial Surgeons of India

Fig. 50.13 (continued)

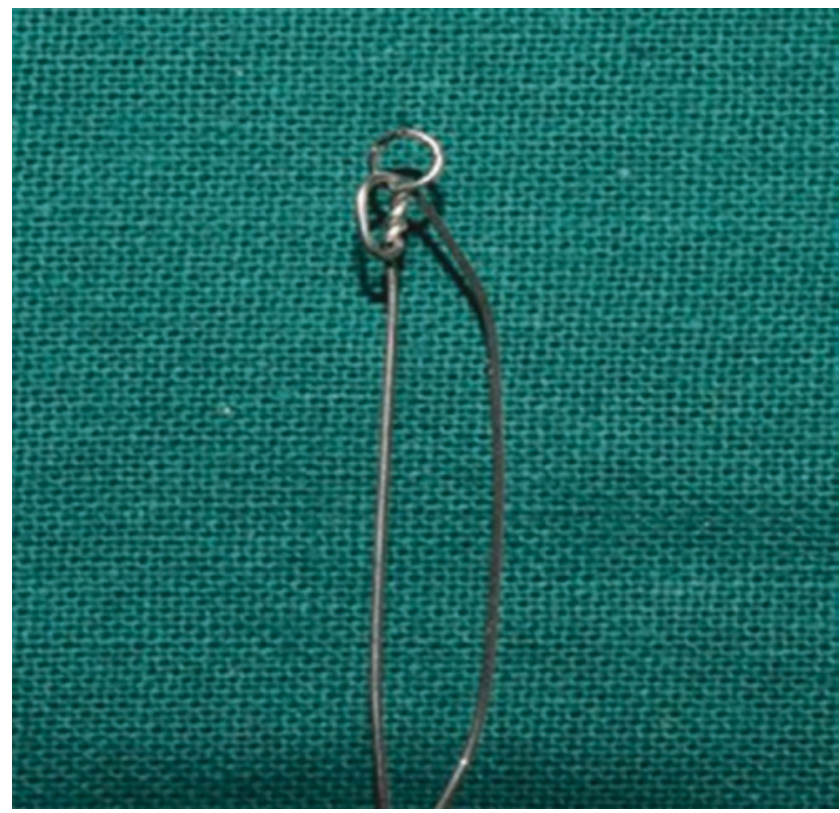

CAssociation of Oral and Maxillofacial Surgeons of India

Fig. 50.14 Shows a modification of Ivy loop ing the wire up to its full length till the midline (Fig. 50.17a). Another wire is passed from the opposite quadrant of the same arch and twisted in a similar manner. Both the ends of this twisted wire are tightened at the midline after reducing the fracture segments (Fig. 50.17b,c). Once anatomic reduction is achieved, additional wires are used to stabilize this long wires by passing around the embrasures of the teeth and looping around the wire segment (Fig. 50.17d) $[26,27]$.

\subsubsection{Obwegeser Wiring $[26,27]$ Technique}

This technique utilizes a $30 \mathrm{~cm}$-long wire that is bent into a continuous "W" arcade form as depicted in Fig. 50.18a. The elevated portion of the arcade acts to form the loop, whereas the depressed portion adapts to the contour of the teeth palatally or lingually. The distal end of the wire arcade is kept long enough so that it will follow the buccal contour of the arch, when it is passed through the interdental space of the most posterior tooth from lingual/palatal to buccal side. The elevated ends of the 

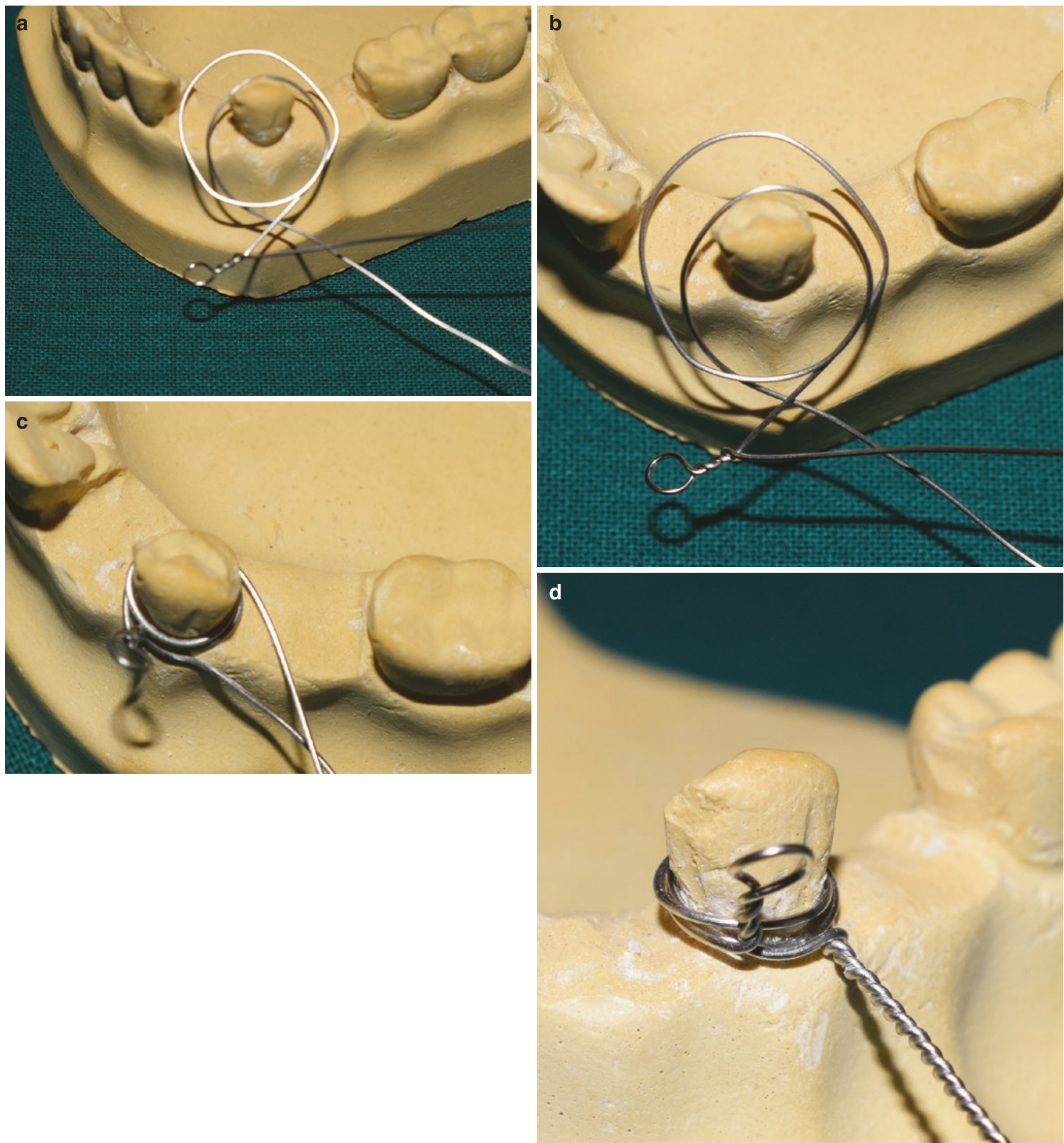

Fig. 50.15 Showing clove hitch technique. (a-c) Single loop is passed around the lone-standing tooth followed by second loop under the first one, pulling both the ends together. (d) Showing clove hitch wiring technique

arcade have a ligature wire attached to it (Fig. 50.18b), which only suffices the arcade to be pulled through to the interdental space to emerge buccally (Fig. $50.18 \mathrm{c}, \mathrm{d}$ ). The long wire is then passed through this loop (Fig. 50.18e), and a modified hemostat is used to twist this wire to form uniform loops. At the mesial end, both the ends of the wire are held together and twisted (Fig. 50.18f). The loops can be used for both elastic traction and wires for securing MMF.

The disadvantage of this technique is that it is very cumbersome and adequate expertise is needed to carry out this technique. 

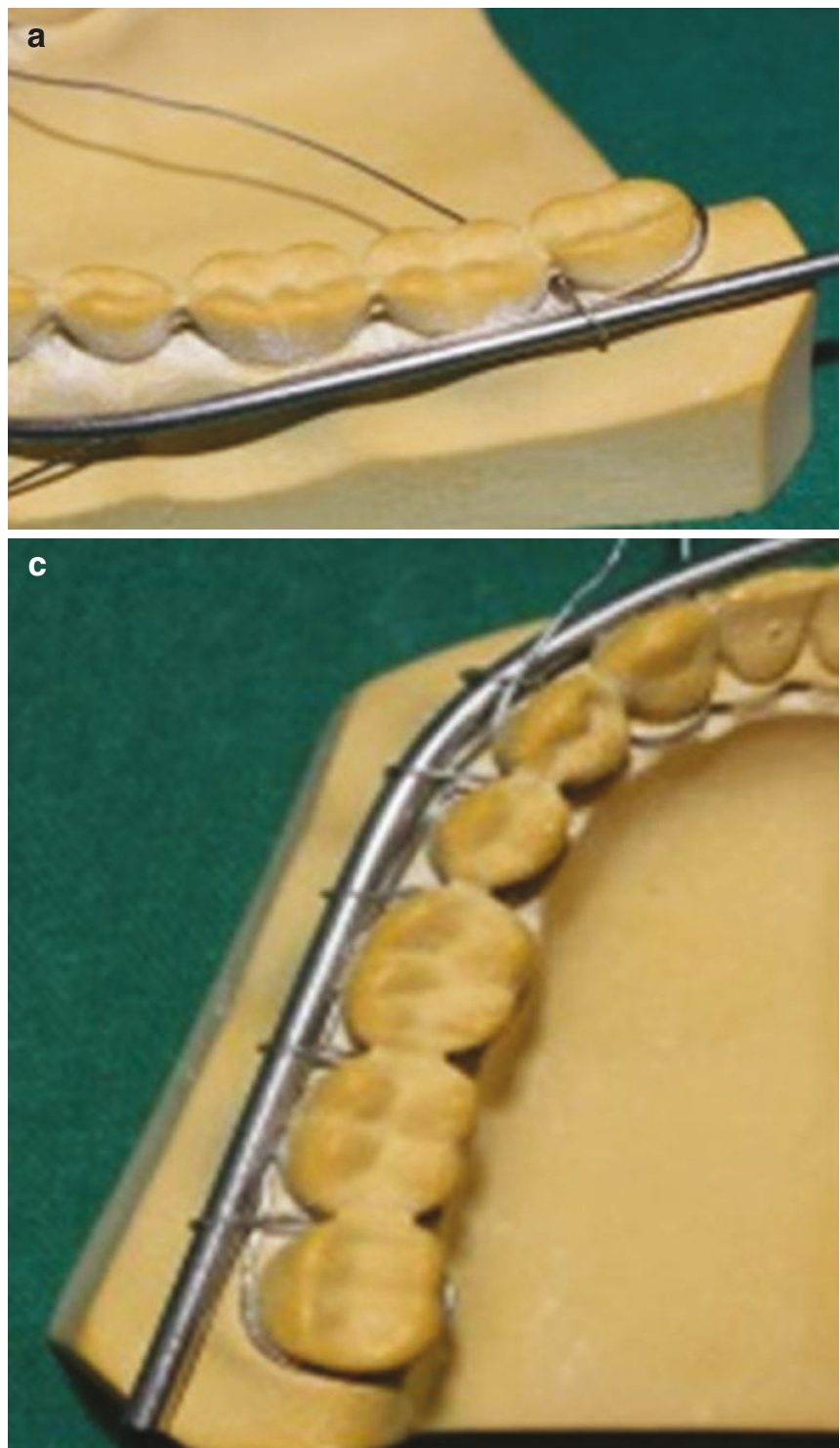

OAssociation of Oral and Maxillofacial Surgeons of India

Fig. 50.16 (a, b) Showing continuous or multiple loop wiring technique, a rod is held parallel to the buccal surface of the teeth, and the wire is passed through the distal most tooth embrasure to emerge buc-
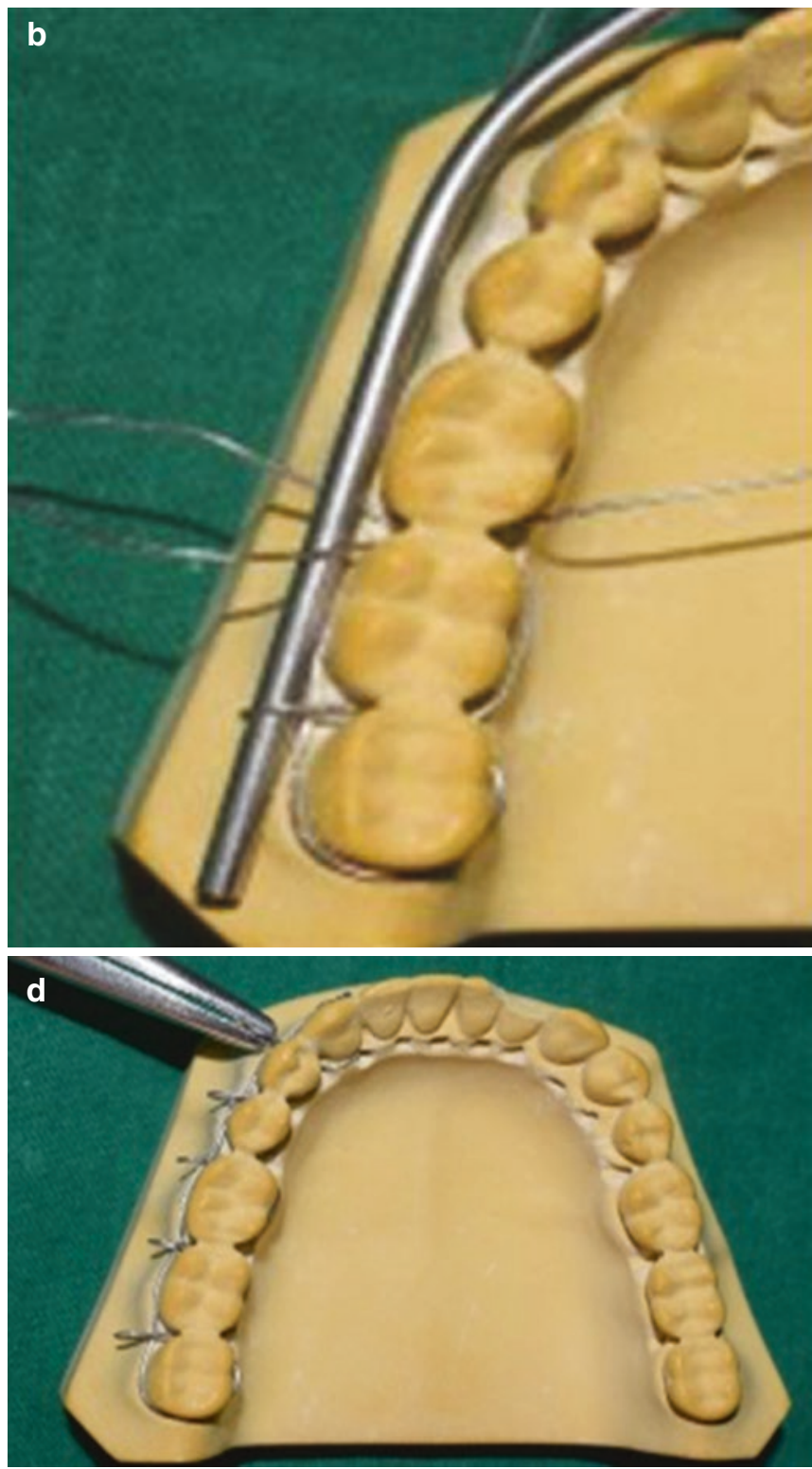

cally and looping around the rod. (c, d) Continuous loops till the midline are made followed by pulling the buccal rod out to achieve loops 

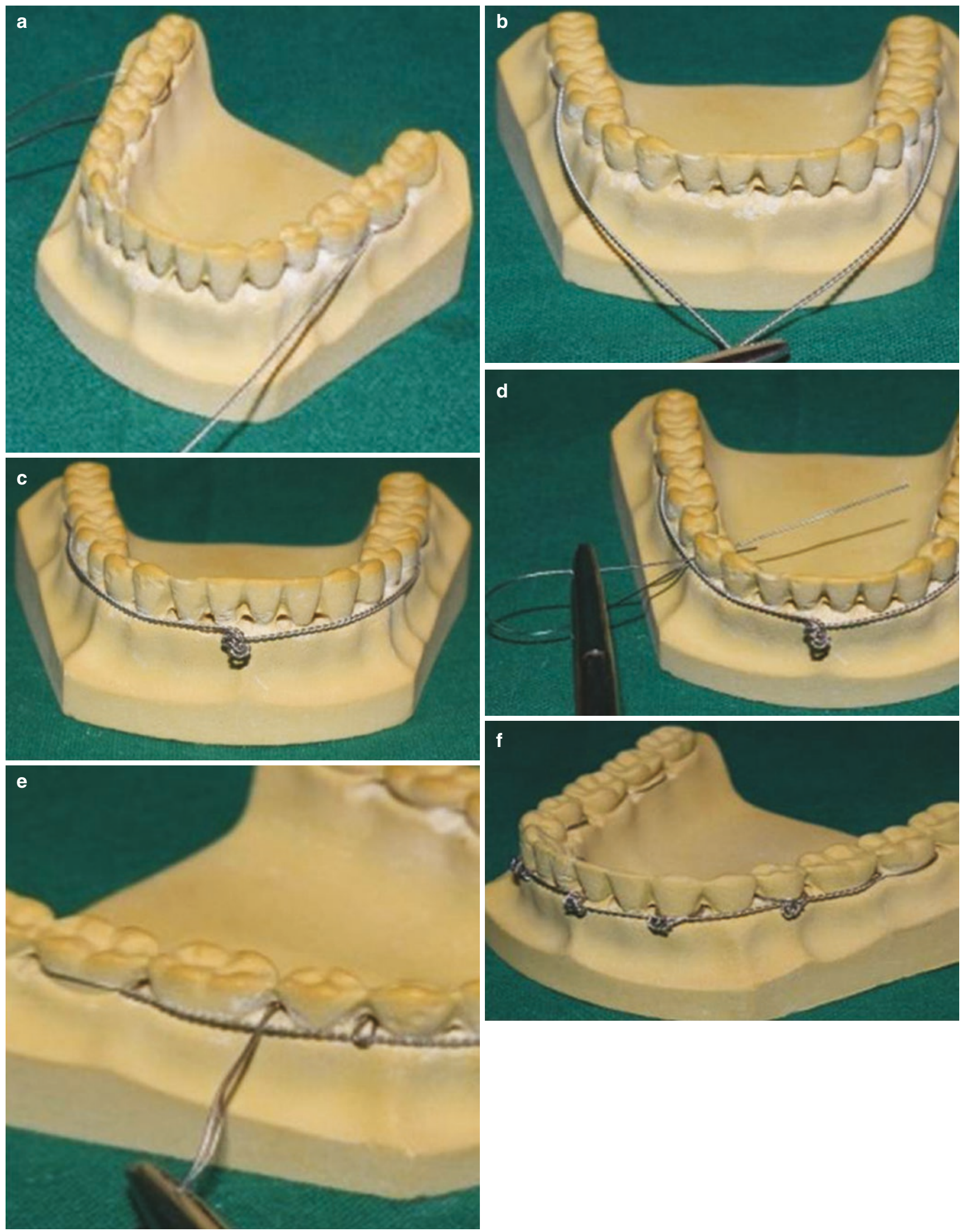

CAssociation of Oral and Maxillofacial Surgeons of India

Fig. 50.17 (a, b) Showing steps in fabrication of Risdon wiring. Distal most tooth is used to loop the wire and twist it. (17c) Showing twisted wire in the midline. (d, e) Showing wires passed through teeth embrasure to loop around the main wire. (f) Alternate method of securing the long wire 

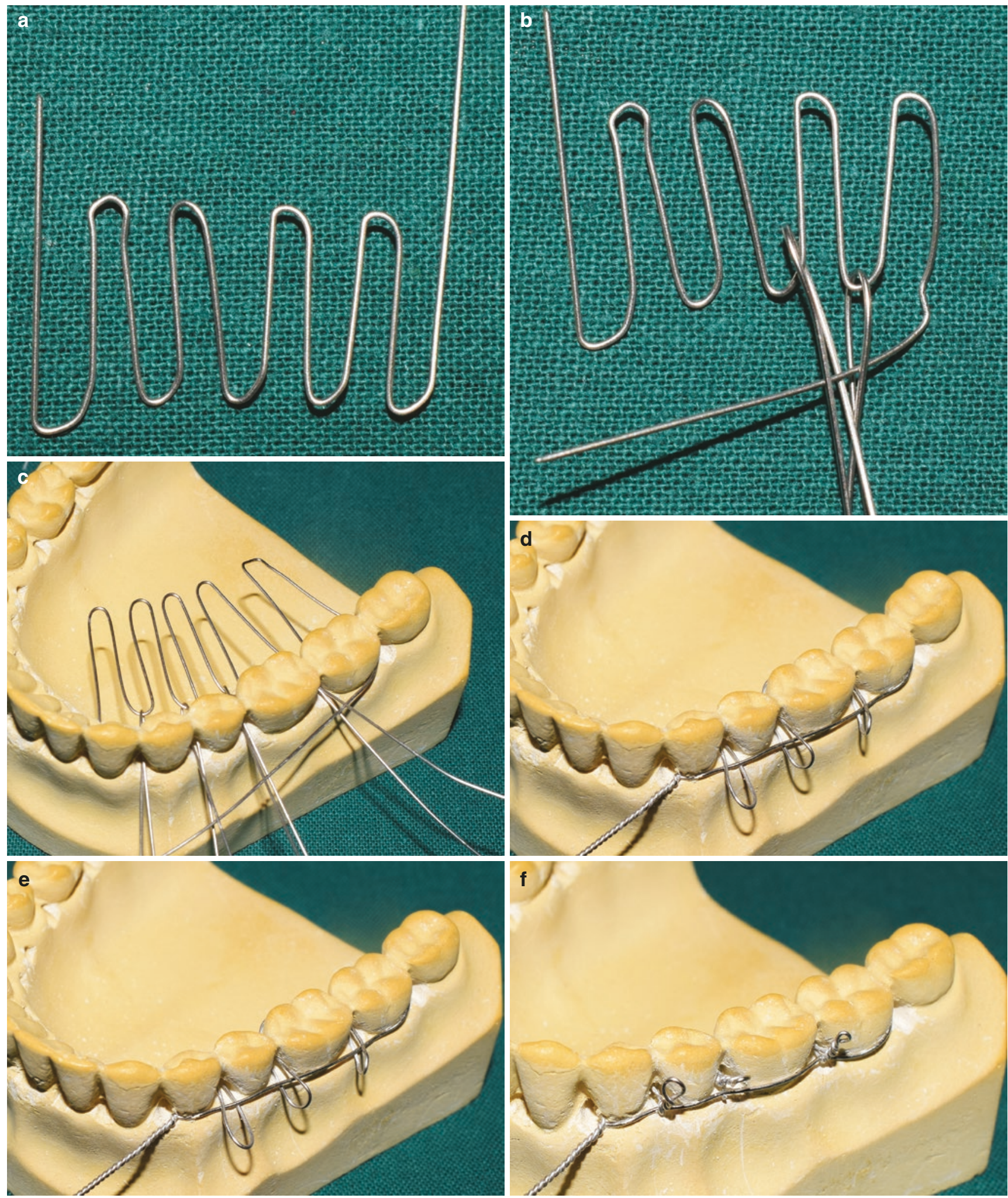

@Association of Oral and Maxillofacial Surgeons of India

Fig. 50.18 (a, b) Showing fabricated W arcade used in Obwegeser technique. (c) Showing ligature wire pulled from the palatal/lingual side to buccal side. (d-f) Showing wire from the distal most end passing though the loops to be twisted at the most medial end of the arcade 


\subsubsection{Arch Bar $[26,27]$ (Video 50.2)}

Commercially available arch bars are usually customized or prefabricated. In most clinical scenarios, prefabricated arch bars like Erich's, Jelenko, Winter's, or Schuchardt's suffice the need for placement.

\section{Indications}

1. Partially edentulous patients where placement of eyelets is not an option due to insufficient number of teeth.

2. The teeth present are far apart making MMF impossible.

3. For alignment of avulsed teeth/displaced dentoalveolar fracture into proper arch form.

4. Reduction and immobilization of dentoalveolar component/ fracture.

5. As a part of integrated treatment for suspension for treatment of maxillofacial fractures.

\section{Technique}

Ideal placement is as close to the interdental spaces, which are then secured with 26 gauge wire. This close placement helps to reduce the arch bar to tooth contact and allows for better adaptability of the arch bar to the arch contour, thereby reducing loosening of arch bar.

In most cases, arch bars are placed from distal of first molar, in one quadrant to the other, maintaining a minimum of two teeth on either side of the fracture line in a dentate patient.

The wire is passed from one interdental space and moved around the tooth to emerge buccally from the other side (Fig. 50.19a). Placement of arch bar by securing it with wire begins (Fig. 50.19b) usually at the midline and proceeds posteriorly or from one end (molar) to another (Fig. 50.19c,d) in order to avoid excess in the center and to achieve uniformity throughout the length of the arch bar. The wires are usually twisted in between the cleats and placed into the interdental space to avoid damage to surrounding soft tissues.

The cleats of the arch bar always face gingivally. MMF is usually secured using 24 gauze wires in box pattern.

Figure $50.19 \mathrm{e}$ is showing a model with upper and lower arch bar placement secured with wires, while Fig. 50.19f is showing a clinical photograph of arch bar placement.

\section{Advantages}

- Easier to manipulate and maintain the intra-arch form.

- Acts as a tension band, resisting forces acting at the alveolar level of the fractured component.

- Multiple vectors can be provided by using elastics and VW cross bracing in reducing the fracture displacement to achieve premorbid occlusion.

\section{Disadvantages}

- One of the major drawbacks is the lack of vertical stop in the anterior region when an arch bar is used. In the posterior region, the opposing teeth act as a vertical stop for occluding teeth which isn't the case with anterior region as the anterior upper teeth may slide over the lower when elastics are stretched between the maxillary and mandibular teeth resulting in extrusion of anterior teeth. This problem can be circumvented, by drilling a hole using a bur in the gingival area apical to the mucogingival junction through the buccal and lingual cortices.

An additional 24 gauze wire is passed from the buccal side of this hole to emerge lingually. The lingual end of the wire is then passed through the interdental space of the tooth to emerge buccally. Both the ends of the wire are then twisted to prevent extrusion.

In the anterior maxilla, anterior nasal spine can be used for additional suspension of the arch bar to prevent downward pull exerted by the elastics.

- Increased operating time.

- High probability of penetrating injuries to the operator.

- Compromised oral hygiene and periodontal tissue damage.

- Post-treatment removal is a traumatic experience.

\section{Complications}

Post reduction of the dentoalveolar fracture, the incisal edges of the fractured teeth bearing segment will remain above the normal occlusal plane. This discrepancy can be corrected by application of apical pressure to this segment through MMF, circummandibular wiring, suspension from ANS, lateral piriform, buttress, or using the eye of the maxillomandibular fixation screw.

\subsection{Maxillomandibular Fixation (MMF) Screws [28] (Video 50.3)}

\subsubsection{Introduction}

A self-drilling or tapping maxillomandibular fixation screws by Arthur et al. [29] in 1989 reduced the intra and postoperative problem associated with the arch bars.

\subsubsection{First Generation}

Screws were modified monocortical self-tapping screws that were kept at $4-5 \mathrm{~mm}$ above the mucosa for subsequent wire 


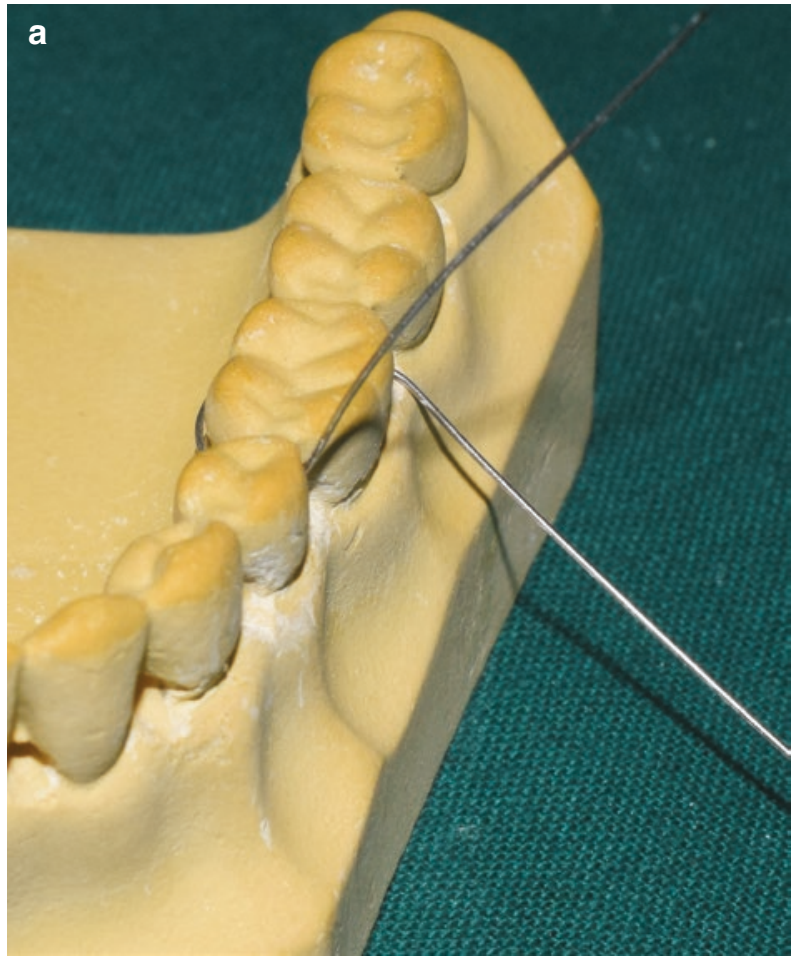

\section{b}

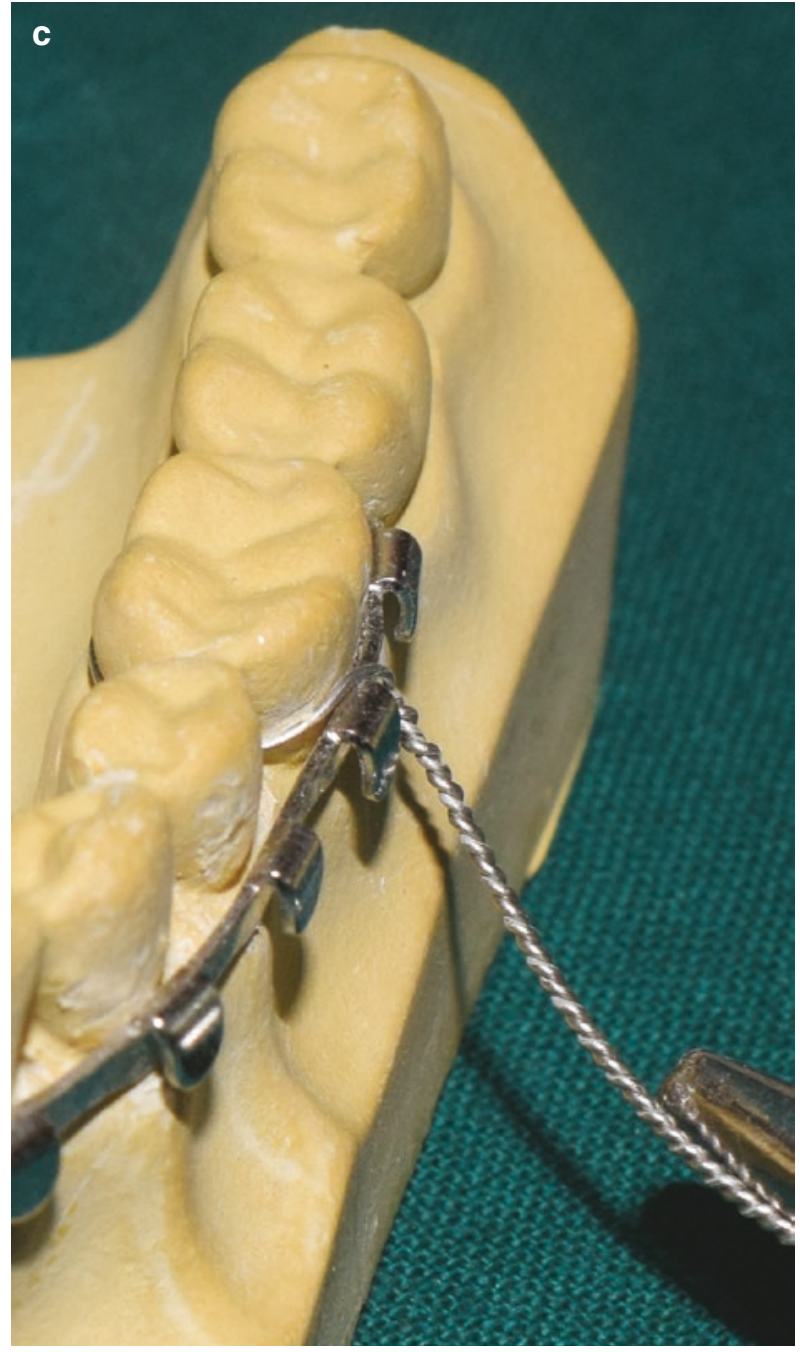

Fig. 50.19 (a-d) Showing technique of arch bar placement. The wire is passed through the embrasure or emerge buccally. The wire on the mesial side of the tooth is passed above the arch bar, where as the distal end wire

is passed below the arch bar to secure it by twisting. (e) Showing a model with upper and lower arch bar placement secured with wires. (f) repre-

senting a clinical photograph of arch bar placement

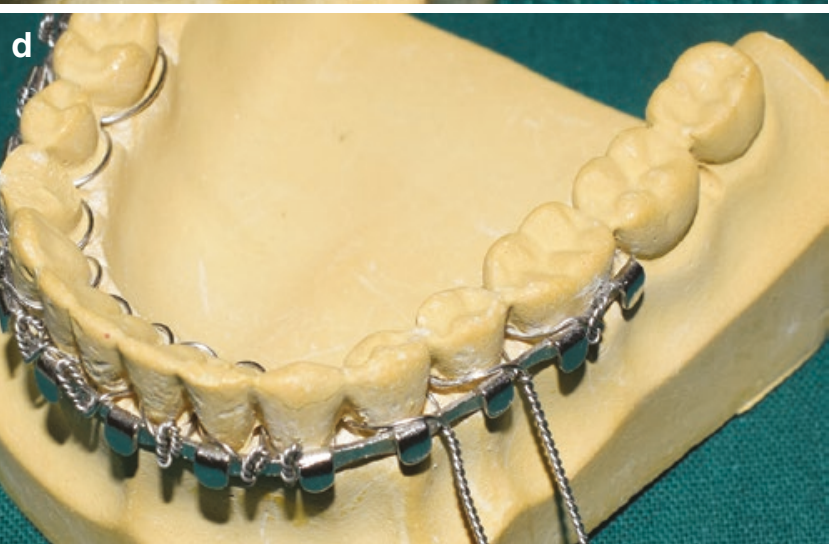




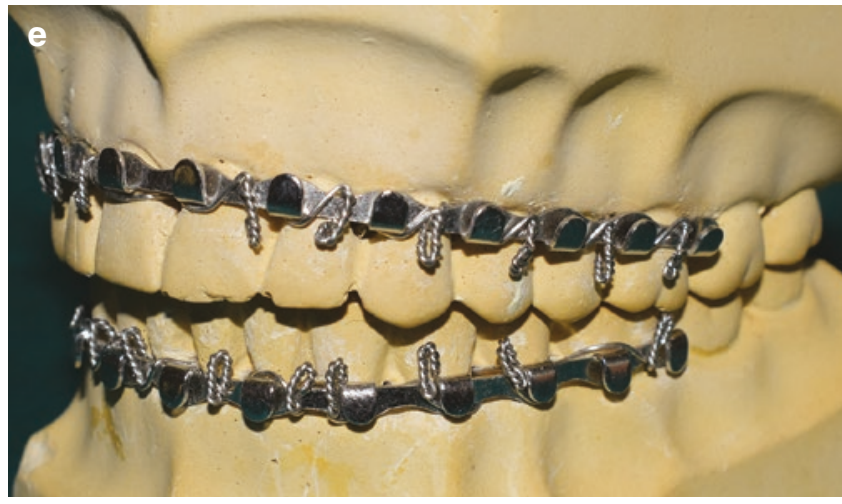

CAssociation of Oral and Maxillofacial Surgeons of India

Fig. 50.19 (continued)

splinting. Surgical preparation of the site needed prior to insertion. They were usually wider in diameter with grooves/ flutes.

\subsubsection{Second Generation}

Screws were self-drilling or self-tapping screws which were spool shaped. The central part of the screw head is perforated with one of two channels perpendicular to its axis aiding in the passage of ligature wires. The top of the screw heads is smoothly finished at the contact zone with overlying mucosa.

Clinician should consider fracture location, dentition, surgical exposure, and the quantity of bone for placement of these IMF screws.

Ideal radiographic images obtained are orthopantomogram and CT axial scan to analyze and plan placement of these screws.

Sites include anterior vestibule and anterolateral (canine and premolar regions) as they provide sufficient bone depth, clearance from adjacent tooth, and easy accessibility. Screws should be placed above the root apices as subapical placement leads to mucosal overgrowth (Fig. 50.20a-d).

Ideally one screw should be placed on either side of the fracture line. However, in severely displaced cases, it is

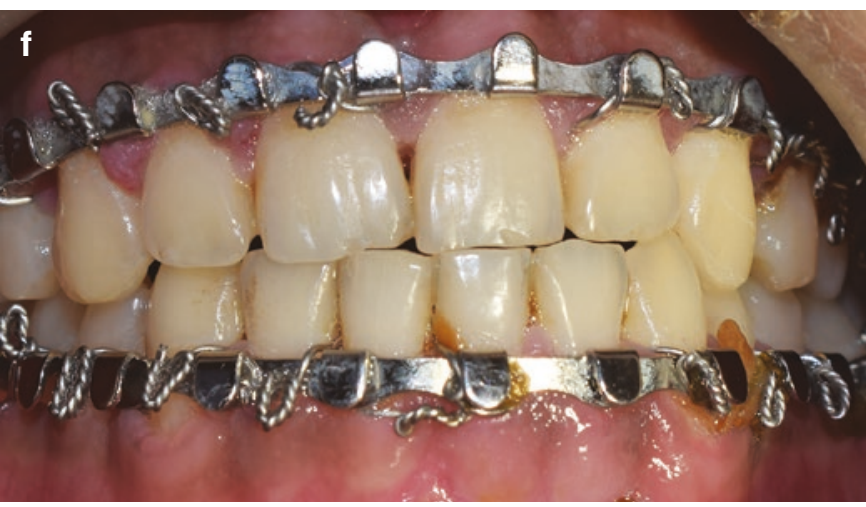

advisable to use multiple screws on either side to achieve vectors in different directions.

\subsubsection{Advantages}

1. Cost-effective

2. Easy placement

3. Lesser operating time

4. Less incidences of penetrating injuries

5. Lesser appliances inside the oral cavity

6. Minimum damage to periodontium

7. Easy maintenance of oral hygiene

\subsubsection{Disadvantages}

1. Iatrogenic damage to tooth roots

2. Root fracture

3. Ingested hardware

4. Screw loosening resulting in malocclusion [30]

5. Pulpal necrosis

6. Root resorption

7. Tooth loss

8. Soft tissue growth over the screw head [31] 

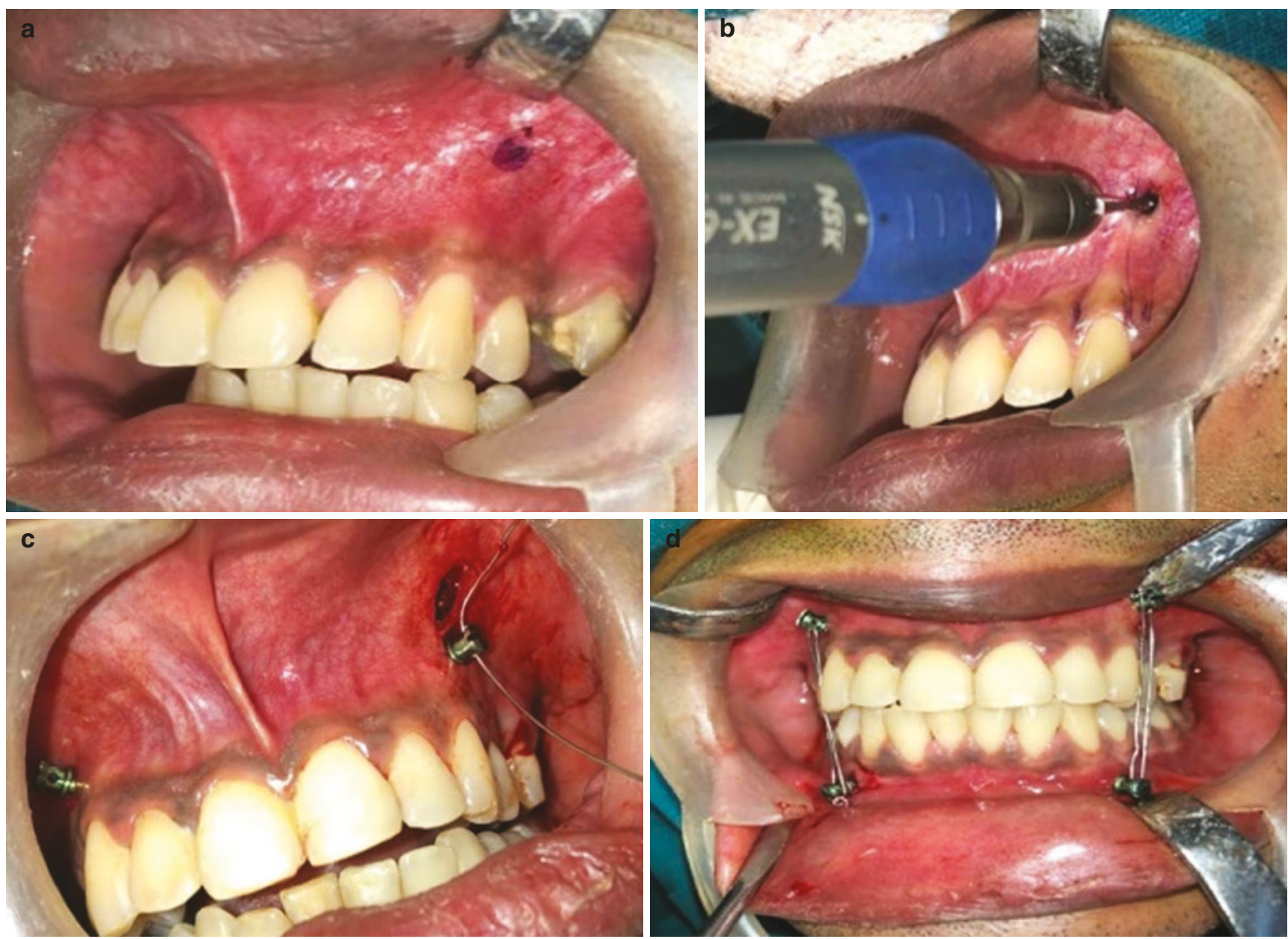

CAssociation of Oral and Maxillofacial Surgeons of India

Fig. 50.20 (a-d) Showing clinical steps in placement of MMF screw. Technique for placement of MMF screws. Marking is done between the root apices followed by drilling and placement of MMF screw, one in

\subsection{Conclusion}

Dentoalveolar fracture mostly involves teeth, soft tissues, and associated bones. With the recent advancements in the field of dentistry, the oral and maxillofacial surgeon can restore the lost form and function of teeth to facilitate the improvement in quality of life in traumaafflicted patients.

Acknowledgments For Figs. 50.1, 50.2, 50.3, 50.4, 50.6, 50.7, 50.8 and 50.9 to Suvy Manuel. each quadrant. The wire is passed through the eye of the opposing arch and secured to achieve intermaxillary fixation

\section{References}

1. Nyardady Z, Orsi E, Nagy K, Olasz L, Nyarady J. Transgingival lag screw osteosynthesis of alveolar process fracture. Int J Oral Maxillofac Surg. 2010;39:779-82.

2. Hale ML. Traumatic injuries of the teeth and alveolar process. In: Kruger textbook of oral and maxillofacial surgery. 6th edn. Jaypee; 1968. p. 357-63.

3. Leathers RD, Gowans RE. Management of alveolar and dental fractures. In: Peterson's principles of oral and maxillofacial surgery. Vol 1, 3rd edn. New York: McGraw Hill; 2004. p. 383-405.

4. Ellis E III, Assael LA. Soft tissue and dentoalveolar injuries. In Contemporary oral and maxillofacial surgery. 2nd edn. Elsevier p. $557-86$ 
5. Sivakumar, Muthu MS. Traumatic injuries of teeth and supporting structures. In Pediatric dentistry principles and practice. 2nd edn. Elsevier. p. 305-26.

6. Pogrel MA, Kahnberg K-E, Andersson L. Traumatic dental injuries. In: Essentials of oral and maxillofacial surgery. 1st edn. Wiley Blackwell; 2014. p. 257-87.

7. Taub PJ, Patel PK, Buchman SR, Cohen MN. Ferraro's fundamentals of maxillofacial surgery. 2nd edn. Springer; 2015. p. 169-73.

8. Margarone JE, Hall R. Management of alveolar and dental fractures. In: Principles of oral and maxillofacial surgery. Vol 1. Philadelphia, PA: J.B. Lippincott; 1992. p. 381-405.

9. Gutmacher Z, Peled E, Norman D, Lin S. Alveolar bone fracture: pathognomonic sign for clinical diagnosis. Open Dent J. 2017;11:8-14.

10. Costello BJ, Martin B. Dentoalveolar trauma. In: Operative oral and maxillofacial surgery. 2nd edn. 2010; p. 475-8.

11. Pagadala S, Tadikonda DC. An overview of classification of dental trauma. IAIM. 2015;2(9):157-64.

12. Faus-Matoses V, Martinez-Vinarta M, Alegre-Domingo T, FausMatoses I, Faus-Liacer VJ. Treatment of multiple traumatized anterior teeth associated with an alveolar bone fracture in a 20 year old patient: a 3 year follow up. J Clin Exp Dent. 2014;6(4):e425-9.

13. Diangelis AJ, Andreasen JO, Ebelesder KA, Kenny DJ, Trope M, et al. Guidelines for the management of traumatic dental injuries: fractures and luxations of permanent teeth. Dent Traumatol 2012;28:2-12.

14. Shulman LE. Transplantation and replantation of teeth. In: Oral and maxillofacial surgery. Vol. 2. St Louis, MO: C V Mosby; 1985. p. $118-42$.

15. Turkistani J, Hanno A. Recent trends in the management of dentoalveolar traumatic injuries to primary and young permanent teeth. Dent Traumatol. 2011;27:46-54.

16. Camp JH. Replantation of teeth following trauma. In: McDonalds RE et al., editors. Current therapy in dentistry. Vol 7. St Louis, MO: Mosby; 1980.

17. Blomlof L, et al. Effect of storage in media with different ion strength and osmolaties in human periodontal ligament cells. Scand J Dent Res. 1981;89:180-7.

18. Bag I, Yildirim S. Effect of avulsion storage media on periodontal ligament fibroblast differentiation. Dent Traumatol. 2017;33(6):458-64.
19. William Cornell R. Management of the avulsed tooth. In: Clinical transplantation in dental specialities. 2nd edn. St Louis, MO: C V Mosby; 1980. p. 89-106.

20. Andreasen JO. Periodontal healing after replantation of avulsed human teeth. Assessment by mobility testing and radiography. Acta Odontol Scand 1975;35:325-35.

21. Abu Samra FM. Dentoalveolar injuries classification-management-biological consequences. J Dent Health Oral Disord Ther 2014;1(4):00025.

22. De Rossi M, De Rossi A, De Queiroz AM, Nelson-Filho P. Management of a complex dentoalveolar trauma: a case report. Braz Dent J. 2009;20(3):259-62.

23. Yamashita D, Cho N. Techniques for maxillomandibular fixation. In: Atlas of oral and maxillofacial surgery. Vol 1.2nd edn. Elsevier; 2016. p. 638-44.

24. McDonald RE, Avery DR, Dean JA, Jones JE. Management of trauma to the teeth and supporting tissues. In: McDonald and Avery's dentistry for the child and adolescent. 9th edn. 2010. p. 403-42.

25. Coletti DP. Contemporary maxillofacial fixation technique. In: Operative oral and maxillofacial surgery. 2nd edn. Medical; 2010. p. 453-61.

26. Williams JL, Rowe NL. Mandibular fractures: closed reduction. In: Rowe and Williams maxillofacial injuries. Vol. 2. 2nd edn. Churchill Livington; 1995. p. 234-66.

27. Marunick MT. Closed reduction and Immobilization of condylar fractures with intermaxillary fixation alternative techniques of Ivy Loops and Molar wafers. In: Atlas of craniofacial trauma. Lippincott Williams and Wilkins; 2012. p. 36-43.

28. Manjrekar S, Joshi A. Use of mandibulo-maxillary fixation screws for the treatment of maxillofacial fractures. Dentistry. 2017;7(12):467.

29. Arthur G, Berardo N. A simplified technique of maxillomandibular fixation. J Oral Maxillofac Surg. 1989;47(11):1234.

30. Rai A et al. Are maxillomandibular fixation screws a better option than Erich arch bars in achieving maxillomandibular fixation? A randomized clinical study. J Oral Maxillofac Surg 2011;69:3015-3018.

31. Rai A et al.Customised screw for intermaxillary fixation of maxillofacial injuries. Br J Oral Maxillofac Surg. 2009;47: 325-26.

Open Access This chapter is licensed under the terms of the Creative Commons Attribution 4.0 International License (http://creativecommons. org/licenses/by/4.0/), which permits use, sharing, adaptation, distribution and reproduction in any medium or format, as long as you give appropriate credit to the original author(s) and the source, provide a link to the Creative Commons license and indicate if changes were made.

The images or other third party material in this chapter are included in the chapter's Creative Commons license, unless indicated otherwise in a credit line to the material. If material is not included in the chapter's Creative Commons license and your intended use is not permitted by statutory regulation or exceeds the permitted use, you will need to obtain permission directly from the copyright holder. 